\title{
Gasdynamic problems for optically inverse media
}

\author{
N. A. Fomin and R. I. Soloukhin \\ Heat and Mass Transfer Institute, Minsk 220728, U.S.S.R. \\ (Reçu le 9 août 1978, révisé le 24 octobre 1978, accepté le 24 octobre 1978)
}

\begin{abstract}
Résumé. - Les caractéristiques ordinaires des lasers gazodynamiques ont été améliorées pour un ordre de grandeur en utilisant le pompage thermique sélectif et le mélange dans l'écoulement supersonique des composants du milieu de travail. Est discutée la simulation en calcul numérique des caractéristiques de base de lasers gazodynamiques à mélange dans l'écoulement supersonique sur les tubes à choc. L'influence du mélange, des perturbations et des phénomènes d'autoabsorption résonnante sur les caractéristiques d'amplification et de puissance dans les lasers gazodynamiques à mélange dans l'écoulement supersonique est particulièrement soulignée.
\end{abstract}

\begin{abstract}
Conventional gasdynamic laser performances have been essentially, to an order-of-magnitude, improved by using selective thermal pumping and downstream mixing of the working medium components. Numerical calculation simulation and shock tube modeling data on mixed flow gasdynamic laser operation and its basic characteristics are summarized and discussed. The influence of flow mixing gasdynamics, wave disturbances and resonance self-absorption effects on the performance, gain and power characteristics of a mixed flow GDL is particularly examined and discussed.
\end{abstract}

1. Introduction. - Among the molecular continuous wave and quasicontinuous flow lasers with the convective exchange of working media, of importance are also the gasdynamic laser systems (GDLs). The attractive feature of such systems with the gasdynamic means of the production of a population inversion is their simplicity of performance and generating a nonequilibrium flow : population inversion of vibrational-rotational levels of, for example, carbon dioxide molecule is obtained simply by rapid adiabatic cooling of the heated $\mathrm{CO}_{2}-\mathrm{N}_{2}-\mathrm{He}\left(\mathrm{H}_{2} \mathrm{O}\right)$ mixture when expanded through a supersonic nozzle due to the differential relaxation between lasing states in the $\mathrm{CO}_{2}$ system [1-4]. The possibility of attaining high gas mixture flow rates and, consequently, high power levels is of importance for gasdynamic ways of the active media removal in the optical cavity. In this case, the population inversion is obtained with the use of purely thermal excitation sources.

One of the most significant disadvantages of the molecular thermally excited systems is its rather low overall efficiency, i.e. the coefficient of the input thermal energy conversion into the coherent radiation energy. This low efficiency $(\eta \lesssim 0.5 \%)$ is due to relatively small fraction of the available vibrational energy partitioned in the total thermal energy stored in the GDL plenum chamber at comparatively low gas temperatures $(T \lesssim 2000 \mathrm{~K})$, and due to significant collisional losses of the vibrational energy in the course of gasdynamic freezing in the supersonicallyexpanding gas flow. The recent results have shown that a substantial efficiency increase in the GDLs can be achieved in the systems with selective excitation of the pumping working mixture component followed by subsequent mixing of the exciting and light emitting gases in a supersonic flow. In these GDLs, a population inversion is produced as a result of rapid mixing of a vibrationally frozen nitrogen flow and a jet of a cold carbon dioxide-helium mixture followed by the near resonance vibrational energy transfer from the excited $\mathrm{N}_{2}$ molecule to the laser upper state of the cold $\mathrm{CO}_{2}$ molecule. In this case, the lower transition level of the $\mathrm{CO}_{2}$ molecule is not populated. The flow of thermally excited and frozen nitrogen is simply produced by a rapid adiabatic expansion of a hot gas in a supersonic nozzle. The resultant inverse medium is then additionally frozen, adiabatically cooled due to a rapid expansion and only afterwards enters the optical cavity. Thus there are two stages of producing population inversions in the selectively excited systems : at the first stage, the required vibrational excitation energy is frozen and stored in nitrogen molecules (vibrational energy carrier), and at the second stage, this energy is transferred to the upper 
laser level of the molecule-emitter, $\mathrm{CO}_{2}$, under secondary freezing conditions. The below estimates and calculations show that as compared to ordinary GDLs, the substantial freezing efficiency and high available vibrational energy concentrations can be attained at both stages of population inversion creation in the systems with selective excitation.

The gasdynamic laser principle based on selective thermal excitation has been first suggested in [5]. The experimental check [6-8] has proved the feasibility of rapid supersonic flow mixing of the working mixture components. However, these mixing schemes and parameters obtained are far from being optimum, hence, the efficiency of these laser systems has appeared to be relatively low. In [8] the value of small-signal optical gain per unit length was only about $g \simeq 0.8 \mathrm{~m}^{-1}$, and the output power in the active medium, $6 \mathrm{~cm}^{3}$ in volume, was only $\sim 60 \mathrm{~W}$, which corresponds to specific laser energy, i.e. to the output power referred to the gas flow rate, of the order of 13-14 $\mathrm{J} / \mathrm{g}$. In calculating the specific available laser energy of the mixed flow. GDLs (MGDLs), only the main energy carrying component, nitrogen, will be taken into account as the energy expenses for active medium heating and excitation are determined by nitrogen (usually the injected emitting component is at a room temperature).

Substantially high characteristics of GDLs with the selective excitation and supersonic mixing have been obtained in [9-13]. In this case mixing occurred in the varying cross-section channel with non-equilibrium flow expansion. With arc heating of nitrogen [12-13] the specific laser beam energy exceeded $20 \mathrm{~J} / \mathrm{g}$, and works [14-17] have shown that with purely thermal nitrogen excitation (by shocked compression and heating) the energy extracted from the MGDL cavity also exceeded $20 \mathrm{~J} / \mathrm{g}$. The experimental data on varying gain have testified the drastic value of $g\left(\sim 3.5 \mathrm{~m}^{-1}\right)$, i.e. of high population inversion by the gasdynamic means. The data on the improvement of the GDLs with selective excitation and mixing in the supersonic flow have been recently obtained in [18]. In the experiments with arc heating source, high gain values $\left(g \sim 5 \mathrm{~m}^{-1}\right)$ have been recorded at specific laser energy being about $50 \mathrm{~J} / \mathrm{g}$. This means that the basic gasdynamic laser characteristics become close to those of electric discharge excitation systems. Several new schemes of mixing and the waste energy extraction as well as numerical and experimental data on amplifying and power MGDL characteristics have been also presented in [19-31] and will be discussed further. On the basis of the numerical and experimental modeling of the main operational processes in GDLs with selective thermal excitation, the present paper defines the most common regularities of these processes and gives recommendations on a proper choice of optimal operational regimes. Experimental data are reported on the effect of gasdynamic phenomena and radiation properties of the medium on the effi- ciency of thermal energy conversion into the energy of stimulated radiation in such systems.

2. Numerical modeling. - 2.1 ESTIMATION OF EFFICIENCY AND THE AVAILABLE VIBRATIONAL ENERGY CONCENTRATION. - As has been pointed out previously, the efficiency and basic output characteristics of a laser system such as gain and specific laser energy greatly depend on a proper choice of the gasdynamic scheme for the production of a population inversion. It is advisable to estimate the limiting parameters and basic characteristics of such systems before discussing the experimental data on different-type GDLs. Figure 1 presents the data on temperature-

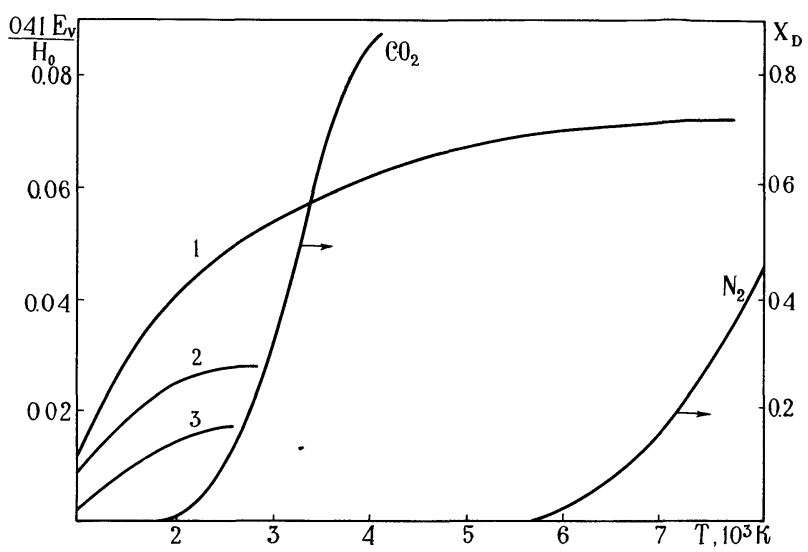

Fig. 1. - The fraction of vibrational energy in the total amount of thermal gas energy divided by the enthalpy, in $(\%)$ that can be converted into coherent radiation energy for nitrogen (1), $\mathrm{CO}_{2}(2)$, $\mathrm{CO}_{2}+4 \mathrm{~N}_{2}+5 \mathrm{He}$ mixture (3), and the dissociation degree of carbon dioxide (4) and nitrogen (5) depending on temperature. The dissociation curves correspond to a gas pressure of $10 \mathrm{~atm}$.

dependent fraction of vibrational energy which can be, in principle, converted into the coherent radiation energy - for pure nitrogen, a $\mathrm{CO}_{2}-\mathrm{N}_{2}$ mixture and for the $v_{3}$-mode of pure $\mathrm{CO}_{2}$. The curves for the temperature-dependent $\mathrm{CO}_{2}$ and nitrogen dissociation degrees are also presented in this figure. It is seen that at high temperatures, the systems with selective nitrogen excitation are advantageous over the variants utilizing premixed $\mathrm{CO}_{2}$ and $\mathrm{N}_{2}$ mixture. Apart from restrictions associated with $\mathrm{CO}_{2}$ dissociation, these advantages are determined by higher vibrational energy concentrations attainable in pure nitrogen at high temperatures. Thus, with increasing initial temperature from $1700 \mathrm{~K}$ to $4000 \mathrm{~K}$, the specific vibrational energy concentration stored in nitrogen increases 5 times. At a nitrogen stagnation temperature of $4000 \mathrm{~K}$, more than $5 \%$ of its total thermal energy is the available vibrational energy that can, in principle, be extracted and converted into the coherent radiation energy (i.e. when the quantum efficiency of the carbon dioxide molecule is taken into account). Note that in the optimum temperature range $(T \sim 1800 \mathrm{~K})$ for conventional GDLs with a 
premixed mixture, the efficiency of the selective excitation scheme is much higher, as there are no waste energy expenditures for helium and carbon dioxide heating.

It is important not only to accumulate the vibrational energy but also to effectively freeze it, and the use of pure nitrogen for these purposes appears to be most appropriate as compared to a $\mathrm{CO}_{2}$-containing

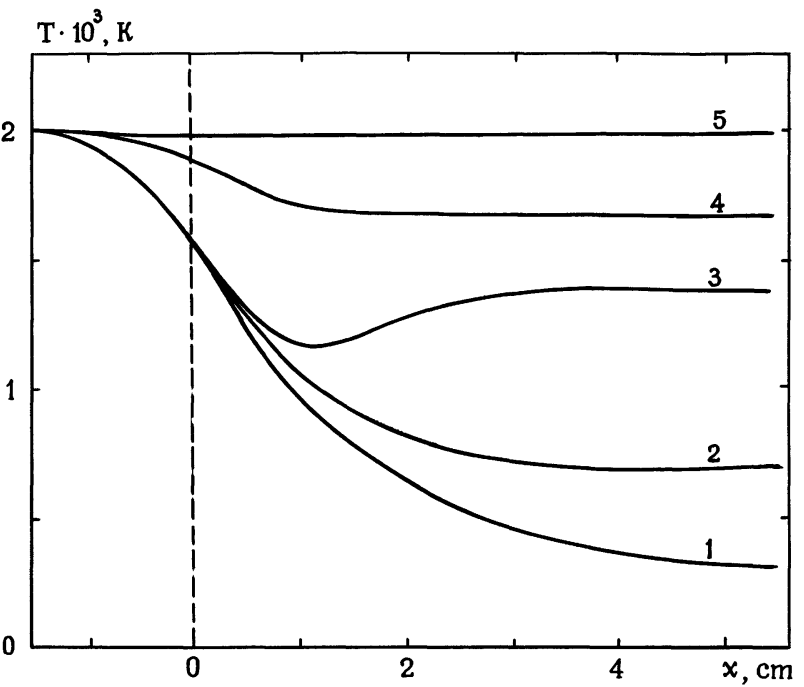

Fig. 2. - Temperature distribution of nitrogen and various $\mathrm{CO}_{2}$ vibrational modes along the nozzle : 1 , translational temperature; 2 , lower $\mathrm{CO}_{2}$ levels ; 3 , upper $\mathrm{CO}_{2}$ levels ; 4 , nitrogen in a conventional-type GDL; 5 , pure nitrogen expansion.

mixture. A comparison of collisional de-excitation times shows that the vibrational relaxation in nitrogen molecules occurs by 2-3 orders slower than in $\mathrm{CO}_{2}$ molecules, therefore, with pure nitrogen expansion the vibrational energy freezes at the very initial stage of gas acceleration and cooling, i.e. it remains close to the initial equilibrium level at plenum temperature. The effect of subsonic freezing of vibrational energy with pure nitrogen expansion in the GDL nozzle illustrates the numerical calculation predictions of a vibrational temperature distribution along the nozzle length which were performed for different gas expansions regimes [16]. These predicted data show that for nitrogen flow in a flat wedge-shaped two-dimensional supersonic nozzle with the throat height of $h_{*}=1 \mathrm{~mm}$ (typical value of $h_{*}$ for premixed gas GDLs), the freezing efficiency of vibrational energy exceeds $90 \%$ at temperatures up to $4000 \mathrm{~K}$. The vibrational energy losses of a premixed $\mathrm{N}_{2}-\mathrm{CO}_{2}$ mixture for optimum parameters in the plenum chamber $\left(T_{0}=1500-2000 \mathrm{~K}\right)$ are not higher than $40-50 \%$, mainly, due to thermalization in the nozzle throat flow region. Low rates of collisional deactivation for nitrogen vibrational states allows the increase of the nozzle throat above $1 \mathrm{~mm}$, thereby increasing the dimensions of a single nozzle in an array of two-dimensional supersonic nozzles thus facilitating the manufacture and assemblage of the GDL nozzle system.

Figure 3 presents the limiting estimates for frozen vibrational energy density which can be, in principle, converted into laser energy (available energy) for GDLs with selective excitation. It follows from these data that selective excitation and freezing of nitrogen only allow more than one-order of magnitude increase in the available energy concentration up to $200 \mathrm{~J} / \mathrm{g}$.

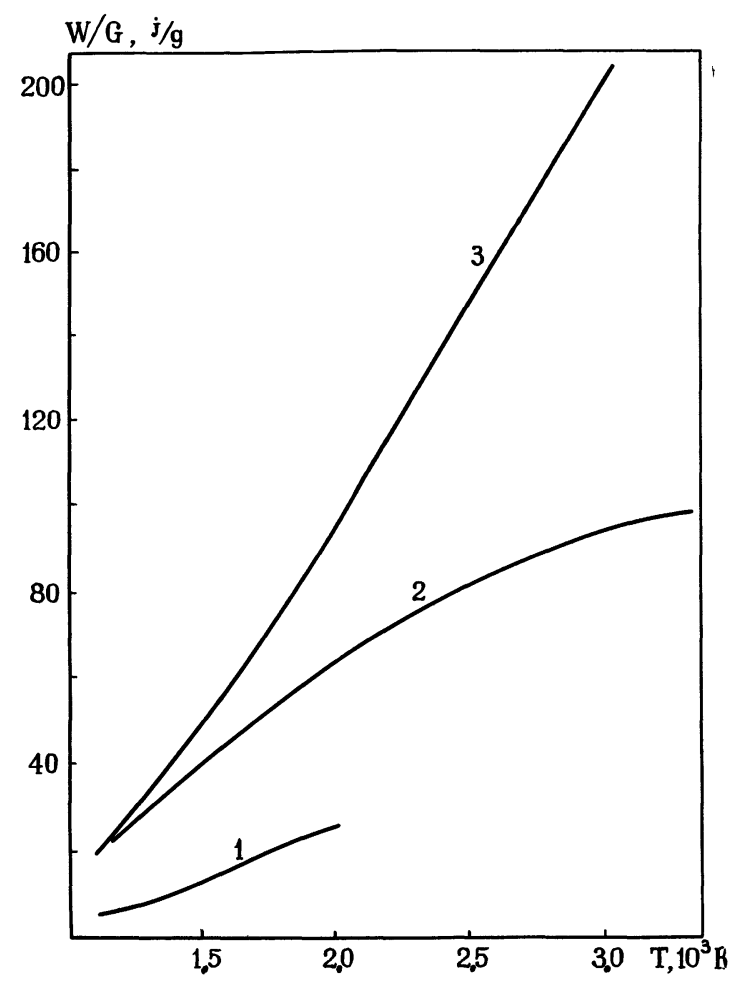

Fig. 3. - Limiting estimates of the available specific laser energy versus temperature : 1 , conventional GDL with a premixed $\mathrm{CO}_{2}+4 \mathrm{~N}_{2}+5 \mathrm{He}$ system; 2, 3, MGDL. Curve 2 takes into account only the available energy at the first vibrational level of $\mathrm{N}_{2}$.

At the second stage of the population inversion creation in GDLs, it is necessary to transfer vibrational excitation from nitrogen to carbon dioxide with minimum losses due to the collisional deactivation. Figure 4 gives the comparative data on vibrational relaxation time (V-T) of the laser upper level of $\mathrm{CO}_{2}-\tau_{3}$ and on vibrational excitation transfer-time (V-V) from $\mathrm{N}_{2}$ to $\mathrm{CO}_{2}-\tau_{34}$ [34]. These data show that the most favourable temperature range at the second stage of the population inversion creation is $300-500 \mathrm{~K}$. In this temperature range, the value of $\tau_{34}$ is more than one order of magnitude smaller than $\tau_{3}$, and the numerical data for typical GDL parameters illustrate that at a distance of about $20 \mathrm{~mm}$, the vibrational energy of nitrogen can be transferred to the laser upper state of the $\mathrm{CO}_{2}$ molecule, practically, without losses.

2.2 ChOICE OF THE MIXING ReGIME. - Cooling of nitrogen, from $3-3.5 \times 10^{3} \mathrm{~K}$ to $300-500 \mathrm{~K}$, is 


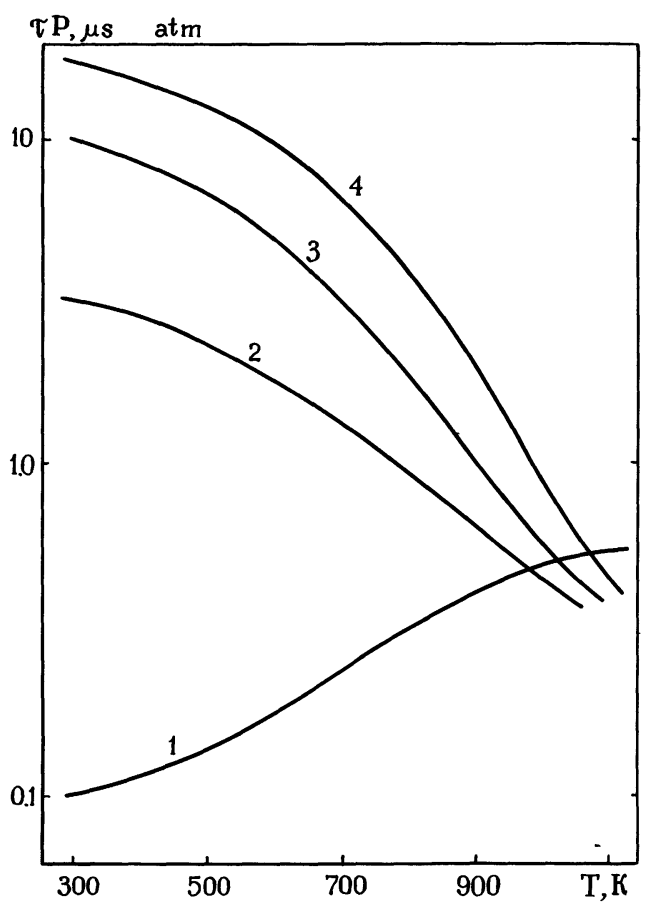

Fig. 4. - Temperature dependences of the vibrational relaxation time, (V-V)-transfer from $\mathrm{N}_{2}$ molecule to the upper laser level in $\mathrm{CO}_{2}(1)$ and relaxation time of the $\mathrm{CO}_{2}$ upper laser level at collision with $\mathrm{He}(2), \mathrm{CO}_{2}(3)$ and $\mathrm{N}_{2}(4)$.

accomplished with gas acceleration expansion up to the Mach numbers of 5-6. The injection at these Mach numbers can be accompanied with certain undesirable gasdynamic phenomena, e.g. shock heating in the supersonic flow stagnation areas. These phenomena as well as the vibrational energy losses and subsequent gain decreases can be substantially reduced by a proper choice of the gasdynamic mixing scheme. So, simple estimations can be made of the thermodynamic mixing parameters using mass, momentum and energy conservation laws. These estimates can be employed particularly to optimize gasdynamic regimes in the injection region.

Different schemes are utilized to inject carbon dioxide into the supersonic hot nitrogen flow (Fig. 5). In the A-variant, gas mixing occurs in the formed supersonic cocurrent flows. This mixing scheme has been employed in [8,17, 19-20, 23-25]. The lower part in figure 5A shows that with flow mixing, an additional expansion can be caused by the finite thickness of the nozzle cut. For example, in [23] 477 jets were utilized in the $\left(20 \times 280 \mathrm{~mm}^{2}\right)$ channel to create the population inversion in the MGDL. The total exit cross-section of 159 nozzles for the carbon dioxide was $\sim 556 \mathrm{~mm}^{2}$, while that of 318 nozzles supplying nitrogen was $3060 \mathrm{~mm}^{2}$. A comparison of the exit cross-sections with the cavity cross-section shows that the latter is 1.55 times greater, which means that the flowing and mixing jets undergo an additional expansion in the screen-nozzle design [23].

In figure $5 \mathrm{~B}$ the secondary flow is injected through
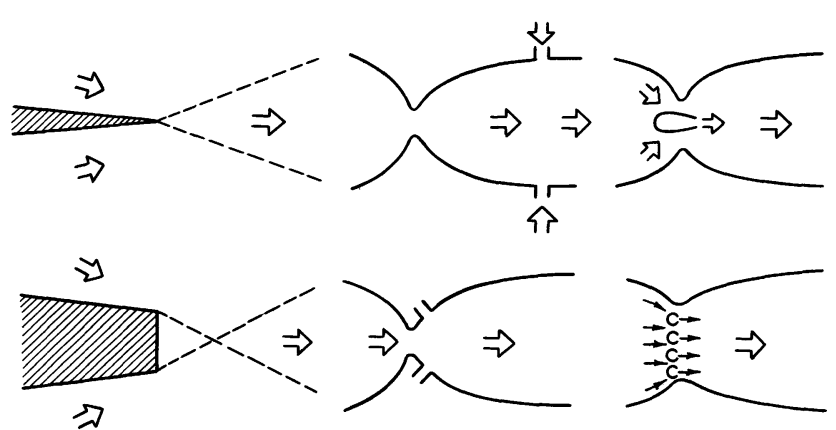

Fig. 5. - Typical flow models in GDLs with selective excitation and mixing in the supersonic flow : A, developed supersonic jets; $B$, injection through the slots in the side walls of the nozzle with additional expansion (lower part of the figure); C, injection into the central part of the nozzle using several inserts with subsequent expansion.

the nozzle side walls. This injection variant (the upper part of Fig. 5B) has been employed in the first constructions of MGDLs [8]. However, considerable stagnation and temperature recovery in this mixing scheme makes it impossible to obtain high population inversion characteristics of working media. At the same time, the scheme (Fig. 5B, lower part) appears to be more effective. The system sensitivity to the choice of a gas-dynamic flow mixing scheme illustrates the results of numerical calculation modeling of turbulentmixing performed in [28] with the use of a PrandtlPatankar-Spalding approach. The limiting values of the small-signal gain in this model (with no gas expansion after mixing) are about $0.5 \mathrm{~m}^{-1}$ (experimental ones [8] are $0.8 \mathrm{~m}^{-1}$ ), while the same predictions give the gain up to $5 \mathrm{~m}^{-1}$ in the case of additional expansion in the mixing region. This has been experimentally proved in [12-13], [18] using the arc heating of nitrogen and by the results of [10-11, 14-17], where nitrogen is excited by purely thermal means, in shock tube powered gasdynamic lasers.

In figure $5 \mathrm{C}$, an additional expansion also occurs behind an injection point, and injection is performed through the central nozzle inserts. Figure 5C, upper part, illustrates the mixing schemes as taken in [10-13], while the lower part of this figure presents those from [16, 17, 27], $\mathrm{CO}_{2}$ and helium mixture being injected through a number of slotted pipes near the nozzle throat. In this version, the primary step of nitrogen freezing is realized with nitrogen expansion between the pipes.

In all these schemes the thermo-gasdynamic parameters vary in the mixing region. A detailed description of the gas parameter distributions in the mixing region involves great difficulties associated with the statement of the problem and with the time consuming numerical calculations of the flow features even in the simple geometry channels. However, the finite state of a medium after mixing can be easily estimated using 
the ordinary equations for mass, momentum and energy conservation.

Assuming an instant mixing of the main (indiced 1) and injected (2) flows, one obtains the resulting equilibrium gas flow parameters (3) as shown in figures 6 and 7 where, similarly to [23], the solutions
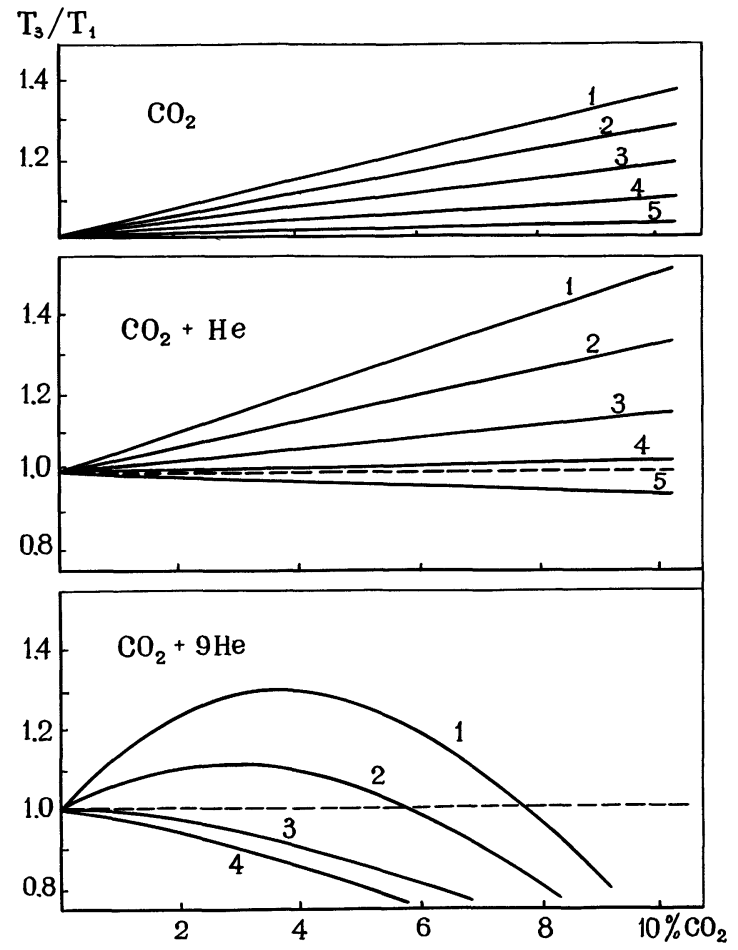

Fig. 6. - Relative temperature variation of nitrogen mixed with additional amount of gas with $\mathrm{CO}_{2}$ concentration in the mixed flow. The main flow Mach number is $M=5$. The numbers at the curves correspond to the Mach number for the secondary flow, $M_{2}$. A stagnation temperature of the secondary flow has been taken from [23] : 1, $T=300 \mathrm{~K} ; 2, T_{2}=390 \mathrm{~K} ; 3, T_{2}=575 \mathrm{~K}$; $4, T_{2}=830 \mathrm{~K} ; 5, T_{2}=1160 \mathrm{~K}$. The injected gas composition is shown in the figure. The plenum temperature is $2000 \mathrm{~K}$. The dashed line denotes isothermal mixing.

are presented as the relationship between the given flow temperature and the required carbon dioxide concentration in the fully mixed flow. In the calculations, only the vibrational nitrogen temperature was assumed frozen at a level of the plenum temperature before and after mixing. It is instructive and advisable to present the results in this way, as it illustrates certain advantages of MGDLs.

In premixed GDLs carbon dioxide concentration in a mixture is not an arbitrary parameter and its choice is determined by relaxational kinetics. Thus, for typical GDL pressures and temperatures of $P=10 \mathrm{~atm}$ and $T=1800 \mathrm{~K}$ the optimum concentration of $\mathrm{CO}_{2}$ molecule is about $10 \%$ and the gain of about $1 \mathrm{~m}^{-1}$. A further increase in carbon dioxide concentration does not bring about gain growth with relaxational losses. On the contrary, with increasing stagnation
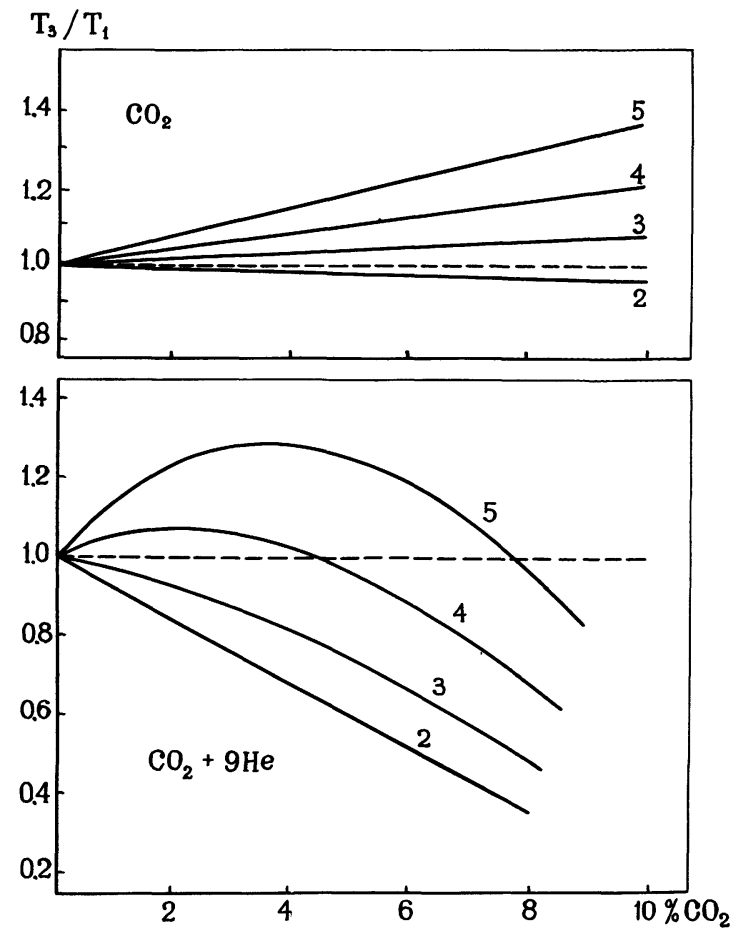

Fig. 7. - The same as in figure 6 but for the Mach number for the secondary flow $M_{2}=1\left(T_{2}=300 \mathrm{~K}\right)$. Numbers at the curves show the Mach number for the main flow.

temperature and pressure [35-36], the carbon dioxide molecule concentration in a working mixture should be suitably reduced in premixed GDLs.

Quite different picture is observed in double-stage MGDLs. Indeed, in this case the efficiency of vibrational energy freezing at the first stage is independent of carbon dioxide concentration, and the vibrational energy losses in the mixing region grow much slower with carbon dioxide concentration than in the premixed GDLs (see the numerical results of the next Section). Therefore, in a wide range of operational parameters, gain in the GDLs with selective excitation is directly proportional to $\mathrm{CO}_{2}$ concentration. Hence, in MGDLs the carbon dioxide concentration in a mixed flow is an arbitrary parameter that facilitates optimization of laser generational characteristics in terms of other parameters. For example, in the GDL with a comparatively small cavity length as in [14-17], [24-25] the gain can attain the value of 3-4 $\mathrm{m}^{-1}$ by increasing carbon dioxide concentration to $15-20 \%$, which allows easy optimization of the cavity parameters. However, it should be borne in mind that vibrational energy losses in the mixing region increase due to collisional deactivation. In GDLs with sufficiently large cross-sections, the gain can be reduced to $0.8-1.0 \mathrm{~m}^{-1}$ and, hence, $\mathrm{CO}_{2}$ concentration can be decreased to $5 \%$.

In figures 6 and 7 the calculation data illustrate a plot of flow temperature recovery versus $\mathrm{CO}_{2}$ concentration after mixing for different injected gas compositions. These data show that in the cold (at a room temperature) carbon dioxide injected with the sonic 
speed into the supersonic $(M=5)$ nitrogen flow, substantial temperature recovery is observed in the mixing region. Preheating and subsequent acceleration of the injected gas have been employed to diminish this effect [23]. Thus, with carbon dioxide heating to $1160 \mathrm{~K}$ affected by subsequent expansion and acceleration to the Mach number, $M=5$ (the nozzle exit temperature of $\mathrm{CO}_{2}$ is close to a room temperature) temperature recovery due to a velocity difference decreases to several percents. It is clear that this way of reducing temperature recovery in the mixing region involves additional energy contributions (about $500 \mathrm{~J} / \mathrm{g}$ in $\mathrm{CO}_{2}$ heating to $1160 \mathrm{~K}$ ), therefore, it is of interest to study alternative possibilities of reducing gas heating in the mixing region. The data in figure 7 show that one of the alternatives is the helium addition into the injected flow. This figure also illustrates the predicted results for temperature variations in the mixing flow with different Mach numbers at the point of injection. The injected gas Mach number in this case was unity. During injection of the $\mathrm{CO}_{2}-\mathrm{He}(1: 1)$ mixture the dependences will remain almost similar to those for pure carbon dioxide injection, however, the mixture with the Mach number (3-3.5) is sufficient to reduce the temperature recovery. When the heliumenriched $\mathrm{CO}_{2}+9 \mathrm{He}$ mixture is used, gas heating is not observed for all $\mathrm{CO}_{2}$ concentrations in the fully mixed flow. Thus, at $\left(\mathrm{CO}_{2}\right) \gtrsim 6 \%$ in the mixing region, the gas cooling is possible due to the enthalpy redistribution.

The calculation results in figure 7 show one more way to reduce gas heating in the mixing region due to decreasing Mach number in the nitrogen flow at the point of injection. Thus, with decreasing Mach number $(M=2-3)$ almost isothermal mixing can be expected even with pure carbon dioxide injection (Fig. 8, upper part). In the injected $\mathrm{CO}_{2}+9 \mathrm{He}$ mixture at $M \lesssim 4$ the cooling effect prevails in the whole range of parameters. It should be borne in mind, however that absolute values of finite temperature at small Mach numbers is substantially greater than in ordinary GDLs, which gives a slight difference between the relaxation time of the upper $\mathrm{CO}_{2}$ level and the time of vibrational excitation transfer from a nitrogen molecule to the upper level of a $\mathrm{CO}_{2}$ molecule (Fig. 4). In this case flow mixing should be accompanied by rapid expansion, i.e. secondary acceleration and cooling of a gaseous mixture (the second stage of gasdynamic freezing). A more detailed discussion of the predicted vibrational relaxational energy losses will be made below.

2.3 RElaxational Processes. Vibrational ENERGY LOSSES IN THE MIXING REGION. - The previous section deals with the estimation of the mixed gas parameters when analysing the properties of GDLs with selective excitation, however, the final choice of the optimal GDL parameters can be made only with regard for relaxational processes. As has already
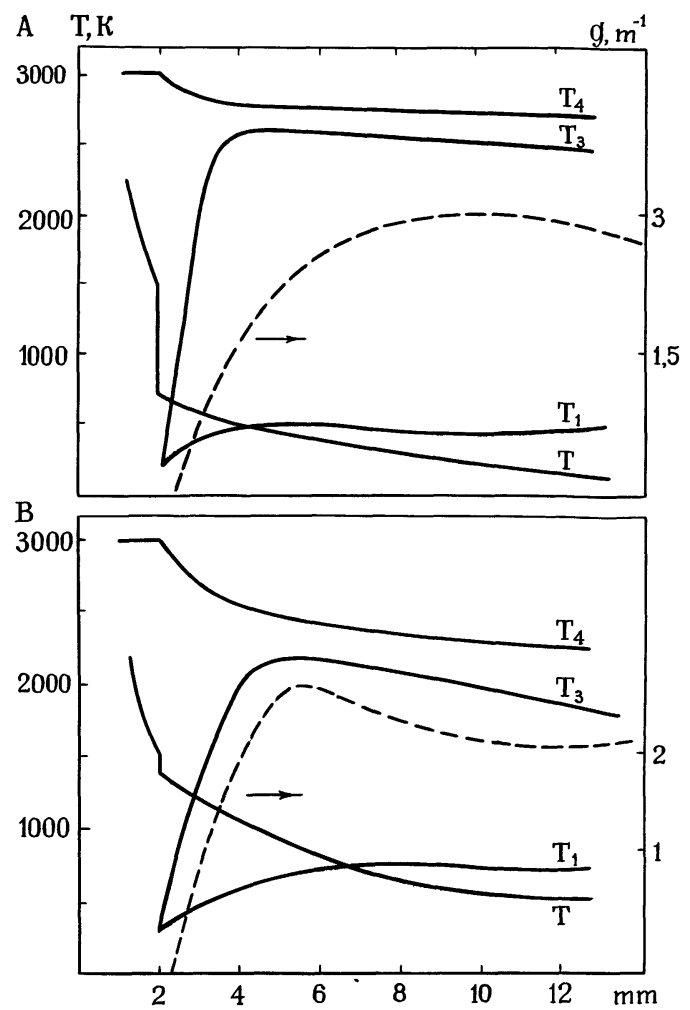

Fig. 8. - Distribution of translational and vibrational temperatures as well as of gain along the nozzle. Plenum temperature and pressure are $3000 \mathrm{~K}$ and $10 \mathrm{~atm}$, respectively; $\mathrm{A}$, injected $\mathrm{CO}_{2}+9 \mathrm{He}$ mixture ; injection pressure is $9 \mathrm{~atm}$; gas composition after mixing $0.055 \mathrm{CO}_{2}+0.452 \mathrm{~N}_{2}+0.493 \mathrm{He} ; \mathrm{B}$, injected $\mathrm{CO}_{2}+$ He mixture, injection pressure is $5 \mathrm{~atm}$; gas composition after mixing: $0.227 \mathrm{CO}_{2}+0.546 \mathrm{~N}_{2}+0.227 \mathrm{He}$.

been mentioned, the effect of relaxation in these systems with selective excitation is substantially less than in conventional GDLs. Nevertheless, in MGDLs an incorrect choice of a flow regime and its gasdynamic parameters may result in significant great losses of vibrational energy due to the collisional relaxation depopulation of the upper and population of the lower laser states. The numerical modeling is useful to analyse these peculiar features in MGDLs.

A simple numerical estimation of the relaxation contribution to the inverted medium characteristics can be performed by solving the relaxation kinetics equations for a fully mixed flow. Such an approach assumes that mixing is fast and vibrational energy losses during mixing itself are small (model for instantaneous mixing). The relaxational kinetics in a medium after mixing is described by the developed fourtemperature model for vibrational relaxation processes, where each type of vibrational mode in the $\mathrm{CO}_{2}$ molecule and states of the $\mathrm{N}_{2}$ molecules are interpreted by the harmonic oscillators, whose energy is defined by dissimilar temperatures of each mode different from the translational-rotational temperature [37-40]. The system of vibrational relaxation equations for the mean number of quanta in the ith mode can be presented as in [40] 
$\frac{\mathrm{d} e_{2}}{\mathrm{~d} t}=\frac{(2 e+1)^{2}}{6 e_{2}^{2}+6 e_{2}+1}\left[\frac{P}{\tau_{2}}\left(\bar{e}_{2}-e_{2}\right)+\frac{3}{2} \varphi_{32}\right]$

$\frac{\mathrm{d} e_{3}}{\mathrm{~d} t}=-\varphi_{32}-\xi_{\mathrm{N}_{2}} \varphi_{34}$

$\frac{\mathrm{d} e_{4}}{\mathrm{~d} t}=\xi_{\mathrm{CO}_{2}} \varphi_{34}+\frac{P}{\tau_{4}}\left(\bar{e}_{4}-e_{4}\right)$

$\varphi_{32}=P K_{32}\left\{e_{3}\left(e_{2}+1\right)^{3}-e_{2}^{3}(e+1) \exp \left[-\frac{\theta_{3}-3 \theta_{2}}{T}\right]\right\}$

$\varphi_{34}=P K_{34}\left\{e_{3}\left(e_{4}+1\right) \exp \left[\frac{\theta_{3}-\theta_{4}}{T}\right]-e_{4}\left(e_{3}+1\right)\right\}$.

Here $e_{\mathrm{i}}=\frac{1}{\exp \theta_{i} / T_{i}-1} ; \theta_{i}$ the characteristic vibrational temperatures of the $i$-th-type vibrations; $P$ the gas pressure; $\tau_{2}$ and $\tau_{4}$ the transfer time of $(\mathrm{V}-\mathrm{T})$ for $\mathrm{CO}_{2}\left(v_{2}\right)$ and $\mathrm{N}_{2}$, respectively; $k_{32}$ and $k_{34}$ the transfer rate constants $\left(\mathrm{V}-\mathrm{V}^{\prime}\right)$. These constants have been chosen as a result of analysing and comparing different experimental data on relaxation kinetics in $\mathrm{CO}_{2}-$ containing mixtures [34] and [41]. The solutions to the equation for vibrational kinetics and to the gasdynamic equations can give the gain, the ultimate specific laser energy and also vibrational energy losses. Typical calculation results [30] are presented in figures 8-10.

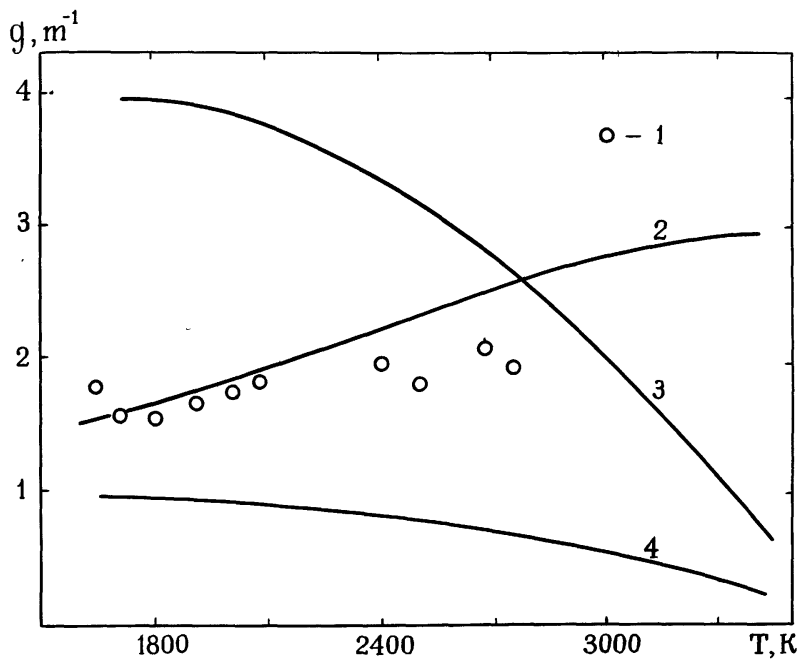

Fig. 9. - A plot of experimental (1) and predicted (2-4) gains versus stagnation temperature. Plenum pressure is $10 \mathrm{~atm}: 1-2$, injection conditions are the same as in figure $8 \mathrm{~A} ; 3,4$, injected $\mathrm{CO}_{2}+\mathrm{He}$ mixture, injection pressure is 5 and $1 \mathrm{~atm}$. The experimental data are taken from [27].

In calculating mixing is assumed to occur in the $\left(\mathrm{CO}_{2}+\mathrm{He}\right)$ injected mixture instantaneously and with no distortion of the main flow pattern (i.e. shock waves do not originate in the flow). Nevertheless, all the variations of the gasdynamic parameters in the injection plane are calculated by the above technique. The vibrational energy losses in pure nitrogen relaxation (up to the $\mathrm{CO}_{2}+\mathrm{He}$ injection point) are

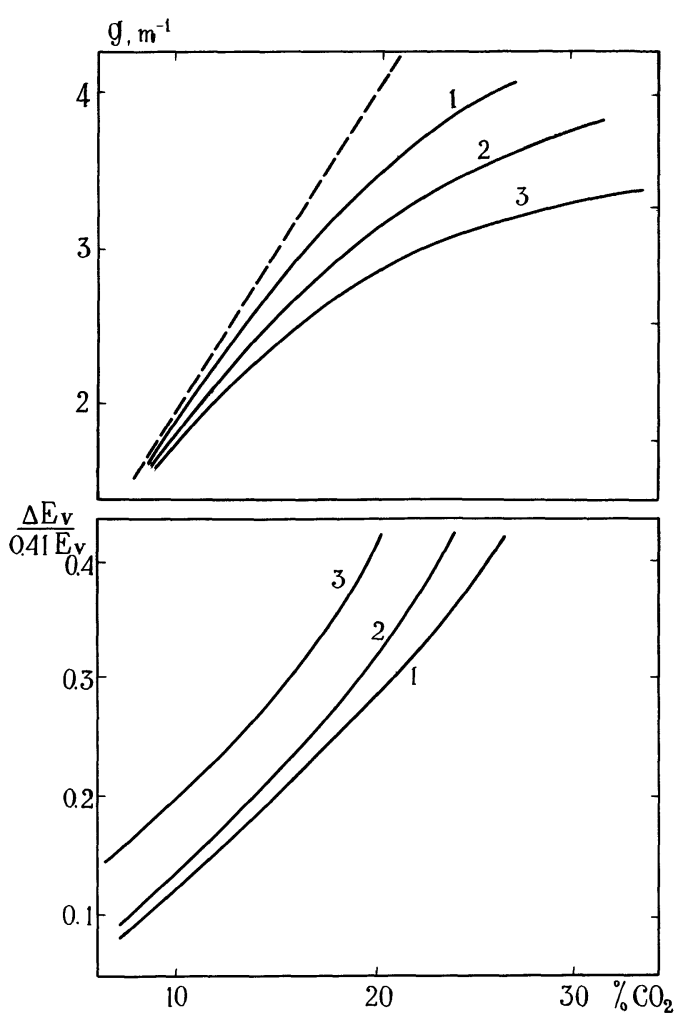

Fig. 10. - Gain and vibrational energy losses behind the point of injection versus per cent content of $\mathrm{CO}_{2}$ in the mixed flow. Losses referred to the total available frozen vibrational energy with regard for quantum efficiency. Plenum temperature is $2000 \mathrm{~K}$, $\mathrm{CO}_{2}+\mathrm{He}$ is the injected mixture; plenum nitrogen pressure (curves 1-3) is 10,10 and $50 \mathrm{~atm}$, respectively; the injection pressure varies from 1 to $7 \mathrm{~atm}$. The dashed line shows a dependence with no regard for vibrational relaxation.

also neglected. This simplification makes it possible to omit integration of the differential equations for transition through the singularity in the vicinity of the nozzle throat. The check measurements made by the time-dependent technique has shown that relaxation losses of the vibrational energy in pure nitrogen cooling through the supersonic nozzle do not exceed $1-3 \%$ of the available vibrational energy. More accurate calculations [42] with regard for inharmonism of nitrogen vibrations have illustrated that losses can be somewhat greater, however, not exceeding $4-7 \%$ for the whole range of GDL parameters with selective excitation and mixing.

Figure 8 presents the predicted data for translational and vibrational temperature distributions along the supersonic nozzle in a mixed flow GDL. It is seen that in the flow behind the injection plane a sharp temperature rise is observed of the asymmetric stretch mode of $\mathrm{CO}_{2}, \mathrm{~T}_{3}$, due to swift near resonance $(\mathrm{V}-\mathrm{V})$ transfer from the first vibrational level of $\mathrm{N}_{2}(v=1)$ to the 001 state of the $\mathrm{CO}_{2}$ molecule. The symmetric stretch mode temperature (in this case the deformation and symmetric stretch modes of $\mathrm{CO}_{2}$ have a common temperature, $T_{1,2}$ ) primarily also shows $1.2-1.5$ fold growth to exceed the translational temperature and 
then slowly changes. Estimations show that small temperature variation of $T_{1.2}$ in this region is determined by almost equal rates of two competing processes, $T_{1,2}$ decreases due to $(\mathrm{V}-\mathrm{T})$ relaxation and its growth due to energy exchange with the asymmetric stretch mode. An increasing level of cooling effect in the injection plane with helium concentration in the injected mixture is also of importance. Thus, for substantial helium concentration in the injected mixture, $\mathrm{CO}_{2}-\mathrm{He}(1-9)$ in figure $8 \mathrm{~A}$, the absolute value of auxiliary cooling is up to $800 \mathrm{~K}$. Figure $8 \mathrm{~B}$ gives the picture of with $\mathrm{CO}_{2}-\mathrm{He}(1: 1)$ mixture during injection. Further gas cooling with its nozzle expansion also occurs more effectively in the first case since the specific heat ratio for a mixture containing a greater amount of helium is higher. Figure 8 shows also the data on the gain distribution along the nozzle under these two mixing regimes.

Typical dependences of the gain on the nitrogen stagnation temperature for different injection regimes are presented in figure 9. The experimental data [27] for the temperature-dependent gain in the MGDLs are also shown in this figure. A comparison of the predicted and experimental data illustrate their good agreement, while at $T \lesssim 2500 \mathrm{~K}$ there has been observed a $20 \%$-difference between the predicted and measured gains. The latter fact is quite satisfactory to make estimations in this simplified processes, as only due to the uncertainty in the value of the rate constants of elementary relaxation stages [39]; the accuracy of the kinetic calculations is limited to $30 \%$. It should be noted, however, that in kinetic calculations of MGDLs the uncertainty of the numerical data is considerably less than in ordinary GDLs since relaxation kinetics in this case is concentrated at the lowtemperature nozzle length (supersonic flow after mixing), and the data on vibrational relaxation rate constants at relatively low temperatures $T \lesssim 1000 \mathrm{~K}$ are more complete.

The predicted dependence of the gain coefficient on the nitrogen stagnation temperature also indicates that the choice of the optimal injected mixture composition strongly depends on the temperature regime : in low-temperature MGDLs $(T \lesssim 2000 \mathrm{~K})$ high gain values are obtained in the injected mixture with a relatively small helium content (high concentration of the emitting gas), while at increasing temperature, the concentration of $\mathrm{CO}_{2}$ should be lessened and helium content should be increased to reduce relaxational losses. This tendency qualitatively fits the experimental study of lasing properties of GDLs with selective thermal excitation and heating for different $\mathrm{CO}_{2}$ and helium amounts in the injected mixture [17].

Figure 10 presents a plot of the gain and relaxational losses of the vibrational energy versus $\mathrm{CO}_{2}$ concentration in the mixed flow. As a result it is possible to choose the optimal carbon dioxide content in the resultant mixture under certain operational conditions and experimental set-ups. It is seen that the gain also increases with $\mathrm{CO}_{2}$; however, in this case the vibrational energy losses behind the mixing plane grow, too. Therefore, the optimum $\mathrm{CO}_{2}$ concentration will be different in laser designs of different cavity sizes : in sufficiently long cavity there is no need to operate at largest gains and energy concentrations, which is caused by considerable collisional losses of the vibrational energy, while in a short cavity the total gain per pass is small and can be compared with mirror and flow resonance absorption losses, hence, to increase gain, the $\mathrm{CO}_{2}$ concentration should be taken higher despite an increase in the relaxational losses of the vibrational energy.

3. Experimental results. - 3.1 SOME SPECIFIC FEATURES OF MODELING OF SHOCK TUBE POWERED GDLS. Application of the shock tubes in high-temperature gasdynamics and chemical physics has become a basis to develop pulse gasdynamic systems that are capable of modeling the operational regimes in the stationary set-up with thermal pumping and with further adiabatic expansion of the working liquid in the nozzle [43-45]. Using the shock wave it is possible to rather fast and uniformly heat the volume of the prepared gas mixture to a temperature of several thousands (or even dozens of thousands) degrees. The shock wave reflection from the closed tube end permits easy modeling of the required stutk gas state in the reservoir of the stationary set-up : the final gas state after double shock compression is uniquely prescribed by the shock wave velocity and initial gas pressure. If a supersonic nozzle with a throat crosssection much smaller than the cross-section of the main tube channel is mounted into the end face of the shock tube, then the final gas state slightly differs from the state behind the reflected plane wave. Alongside with the application of shock tubes for hightemperature gasdynamic modeling in stationary GDLs, they appear to be very significant in the development of the systems with separate thermal excitation and mixing [9-11], [14-17], [24-27].

The use of compression and heating as a source of nitrogen thermal excitation in shock waves has a number of evident advantages, as compared to other ways of thermal pumping due to :

1. the absence of some other types of excitation (electrical, chemical, etc.) apart from pure thermal excitation;

2. the simplicity of the experiments provides an extremely wide range of temperatures, pressures and heated gas flow rates;

3. pulse but quasistationary operational regime of the system makes it possible to avoid application of powerful stationary heaters and cooling devices, and practically eliminates undesirable thermal effects in optics.

The pulsing character of the process and measurement of different parameters in shock tubes require 
a scrutinizing analysis of a transition stage of the working process (i.e. starting regime of the supersonic nozzle) and control of the quasistationary flow. This analysis of modeling gasdynamic properties of the lasers with shock heating has been performed in [46]. Shock wave reflection its pass through the nozzle and development of the quasistationary shocked gas through the nozzle are the complex of complicated unsteady-state and nondimensional gasdynamic processes difficult for a detailed theoretical description. Figure 11 illustrates the diagrams for wave processes

A

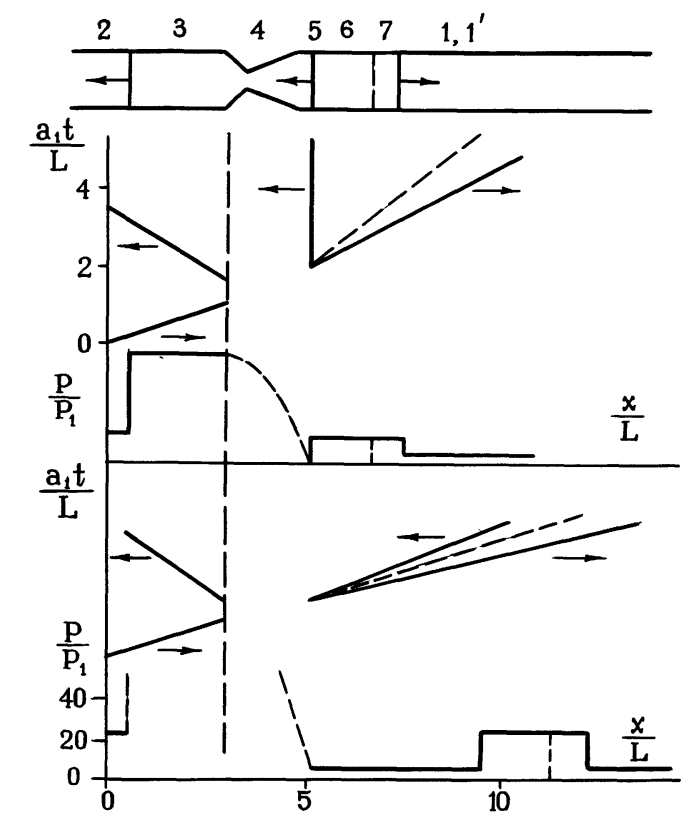

Fig. 11. - Scheme of wave processes with quasistationary expansion in the shock tube for two typical regimes : a, with no diaphragm in front of the nozzle; $b$, with diaphragm at a pressure drop before flow expansion $\left(10^{3}\right)$. The incident Mach number is $M_{1}=3.2$.

in the nozzle mounted at the end face of the shock tube. In predicting such flows the vector polar method [47] is simple and effective and ignores the details of the wave processes in the transition region, and matching of the main gas flow characteristics occurs before and behind the transition region - in the quasistationary approximation. Application of this method to the conditions, typical for gasdynamic laser flows, allows sufficiently accurate calculation of the main flow characteristics : velocity of the incident and reflected shock waves, velocity of gas expansion, wave picture in the expanding section of the nozzle, etc.

In [46] it has been shown that with quasistationary expansion at the nozzle exit, the secondary shock wave is formed, which moves towards the expanding gas flow, then it is stalled downstream with the incoming flow. In this case the speed of the wave stalling from the cavity and, hence, the time of attaining the predicted parameters in the cavity substantially depends on the initial nozzle pressure. The analysis of the wave diagrams presented in figure 11 shows the necessity of preliminary pumping out of the nozzle section to about 1 torr. With the initial nozzle pressure of 0.1-0.05 atm the stalling speed of the secondary shock waves relative to the channel walls is extremely small $\left(u \lesssim 0.1 a_{0}\right.$ where $a_{0}$ is the sound velocity in the plenum chamber), and the starting time depends only on the time of secondary shock wave stalling and may be several milliseconds. In case of the initial nozzle pumping out, the stalling speed of the secondary shock wave is high enough being of (2-4) $a_{0}$ and, consequently, does not limit the time of the quasisteady stage. As the analysis of [48] shows, the total transient time depends mainly on the development in unsteady waves and is between $100-200 \mathrm{~ms}$ for typical GDLs.

3.2 Experimental. - A typical schematic of the experimental set-up with the shock tube for modeling the basic processes and power output characteristics of MGDLs has been described in [14-16]. A conventional shock tube with a rectangular cross-section $\left(3 \times 12 \mathrm{~cm}^{2}\right)$ was employed, the reflected shock pressure being between 5 and $40 \mathrm{~atm}$ at stagnation temperatures from 800 to $7000 \mathrm{~K}$ and mass flow rates of 0.1 to $0.3 \mathrm{~kg} / \mathrm{s}$. In addition to various modes of $\mathrm{CO}_{2}$ injection into an expanding $\mathrm{N}_{2}$ flow (see Fig. 5), a porous wall injection system has been used as shown in figure 12 . There are many advantages of this system

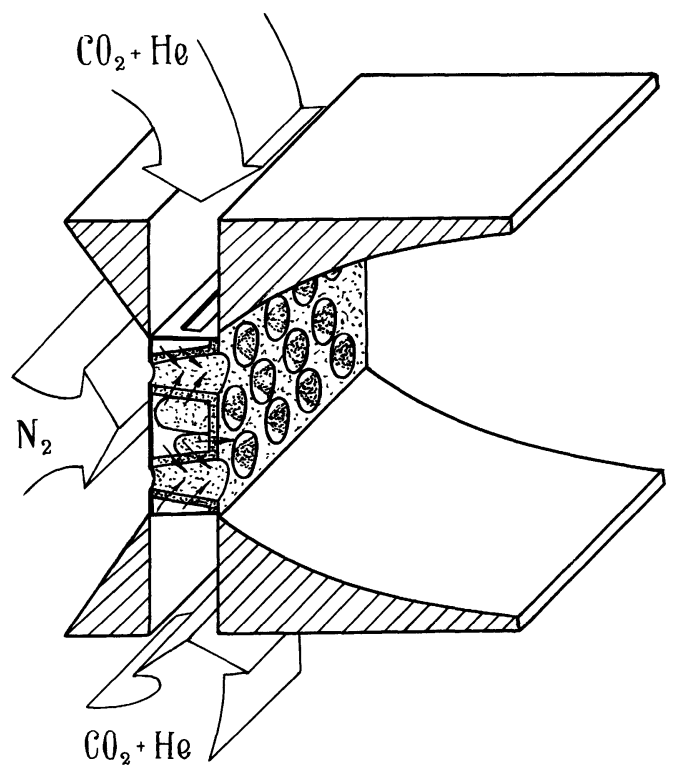

Fig. 12. - Nozzle section of the mixed flow gasdynamic laser. The nitrogen flow is expanding in an array of 15 contoured nozzles $\left(d^{*}=3 \mathrm{~mm}, A / A^{*}=4\right)$. The $\mathrm{CO}_{2}+$ He flow is injected through porous walls, then the flow undergoes additional expansion. The total area ratio is $A / A^{*}=15$.

associated mainly with its dispersivity, lack of gasdynamic disturbances in the gas injection area and homogeneity of the mixture composition and gain distributions across the nozzle flow.

In the lasing regime, a multi-pass (from 3 to 15) four-mirror optical cavity was installed behind the nozzle exit as shown schematically in figure 13 . 


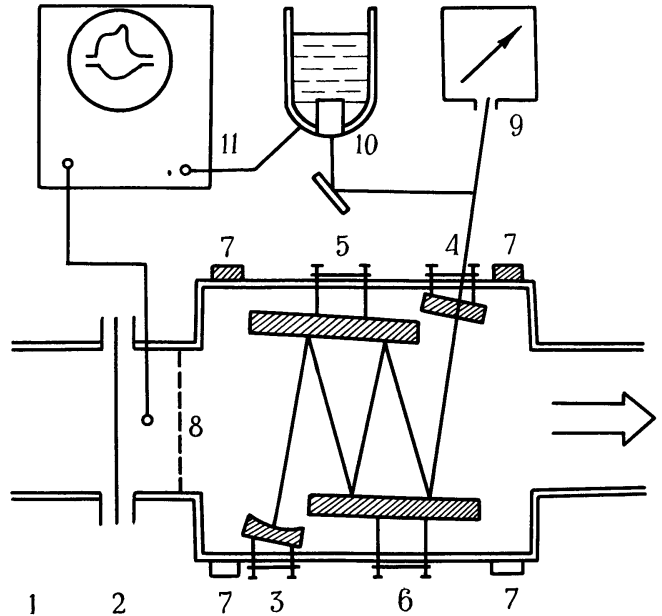

Fig. 13. - Schematic diagram of the multi-pass cavity in a shock tube driven laser [17] : 1 , shock tube; 2 , diaphragm ; 3 , spherical mirror; 4, semi-transparent mirror; 5, 6, flat mirror plates; 7, infrared windows ; 8, nozzle ; 9 , calorimeter ; $10, \mathrm{Ge}-\mathrm{Au}$ detector ; 11, oscilloscope.

The number of passes varied to achieve the prescribed optimum regime corresponding to a given transparency of the output mirror. The mirror was a planeparallel $\mathrm{KCl}$ or $\mathrm{KRS}$ plate with dielectric coating. For the active medium $12 \mathrm{~cm}$ long, the optimum number of passes for the output mirrors was found to be 5 and 11 , respectively.

3.3 OPTICAL QUALITY OF THE FLOW. - With gas injection into the supersonic flow in the GDL nozzle a number of complex non-dimensional gasdynamic phenomena are observed and affect both the starting processes and the behaviour of the steady-state flow itself. One of the most significant characteristics of the optical quality of the flow in the GDL cavity is the distribution and varying behaviour of the expanding gas density. The interferometric measurements made to visualize the flow pattern in the supersonic nozzle flows of the MGDLs show that for two types of the flow, with injection through the side walls and with the central insert used, the processes of achieving steady-state flow in MGDLs have the same structure and sequence as those in ordinary GDLs (Fig. 14). All transient processes practically cease in 0.2-0.4 ms. Flow density fluctuations in the steady-state expansion phase have characteristic times of the order of 1-5 usec and are approximately from $1 / 5$ to $1 / 10$ of the interference fringe in MGDLs, i.e. 3-6\% of the total flow density, thereby a little exceeding the fluctuation level in the premixed gas system (ordinary GDLs). Of special importance is the gasdynamic regularity of the flow entering the cavity, i.e. the absence of shock waves, separated regions, etc.

3.4 GaIn MEASUREMENTS. - An important property of the MGDLs is the gain distribution across the nozzle. It reflects the dynamics of flow mixing and, to a great extent, defines the amount of power in the lasing regime. The experiments [9-11] and the data $[12,13]$ have shown that the mixing intensity
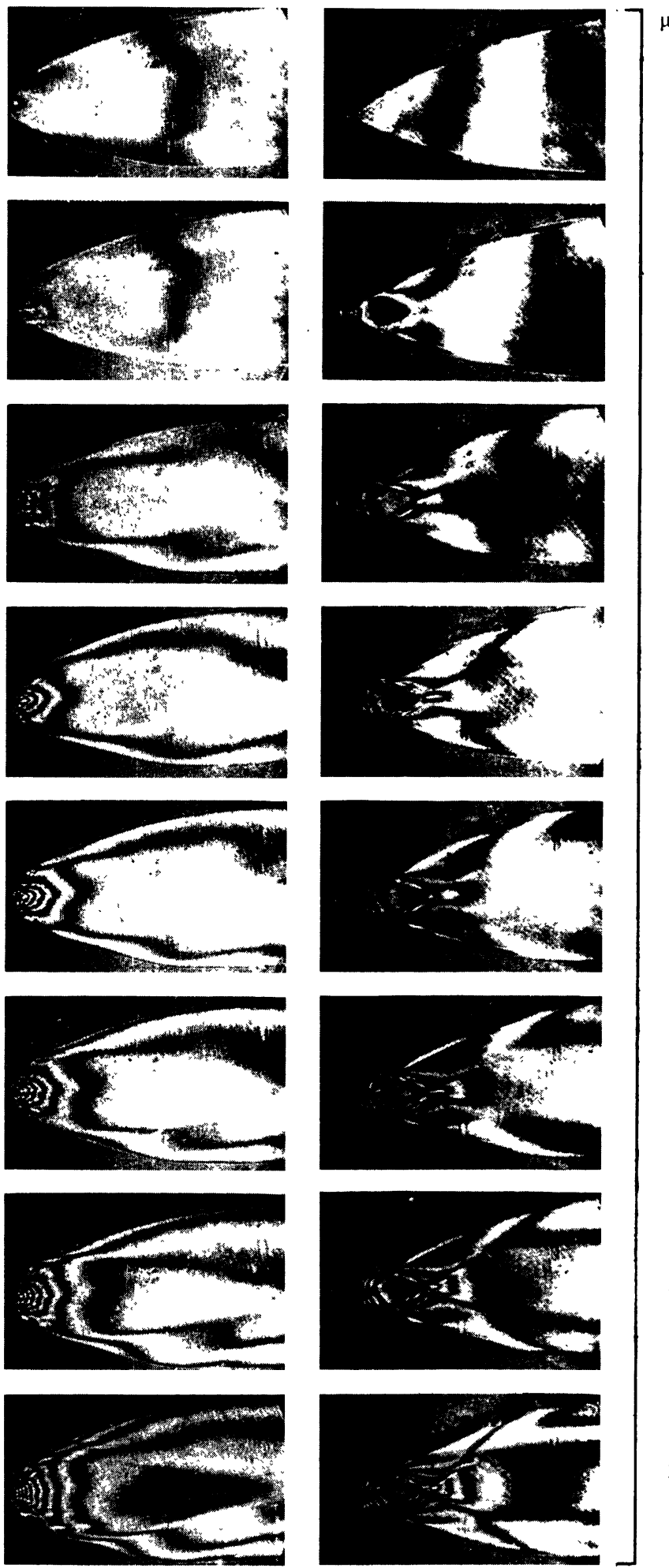

Fig. 14. - Nozzle flow fields with different mixing schemes : 1, injection into the central part of the nozzle ; 2 , injection through the side walls.

with central injection parallel to the main flow is comparatively small, and the gain has its maximum at the nozzle centre and then falls in the wall direction. However, the injection at a small angle to the main 
flow, in the case of the central insert [18], allows substantial enlargement of the inversion area and improvement of a gain distribution across the nozzle. The similar studies of the dependences of the gain in MGDLs on different operational parameters have been also made in [14-17, 23-27].

When injecting the flow through the nozzle sidewall slots, the region of a maximum gain is displaced to the nozzle wall, the gain values being very high $\left(g \sim 3.5 \mathrm{~m}^{-1}\right)$. It should be noted, however, that in this case the gain distribution across the nozzle is not uniform. As is seen from the data in figure 15, a more uniform gain distribution across the nozzle can be obtained with the slotted tubes (Fig. 15C), though the finite values of $g$ are somewhat smaller than those in the variants discussed above. Figure 15D gives the measurements of the gain distribution in the central nozzle section (variant in Fig. 15C). It is seen that in the mixing regime the inversion is observed at a distance of $x<2 l_{*}$, where $l_{*}$ is the injection tube height $(0.8 \mathrm{~cm})$ or $x<20 h_{*}$ where $h_{*}$ is the distance between the tubes.

A
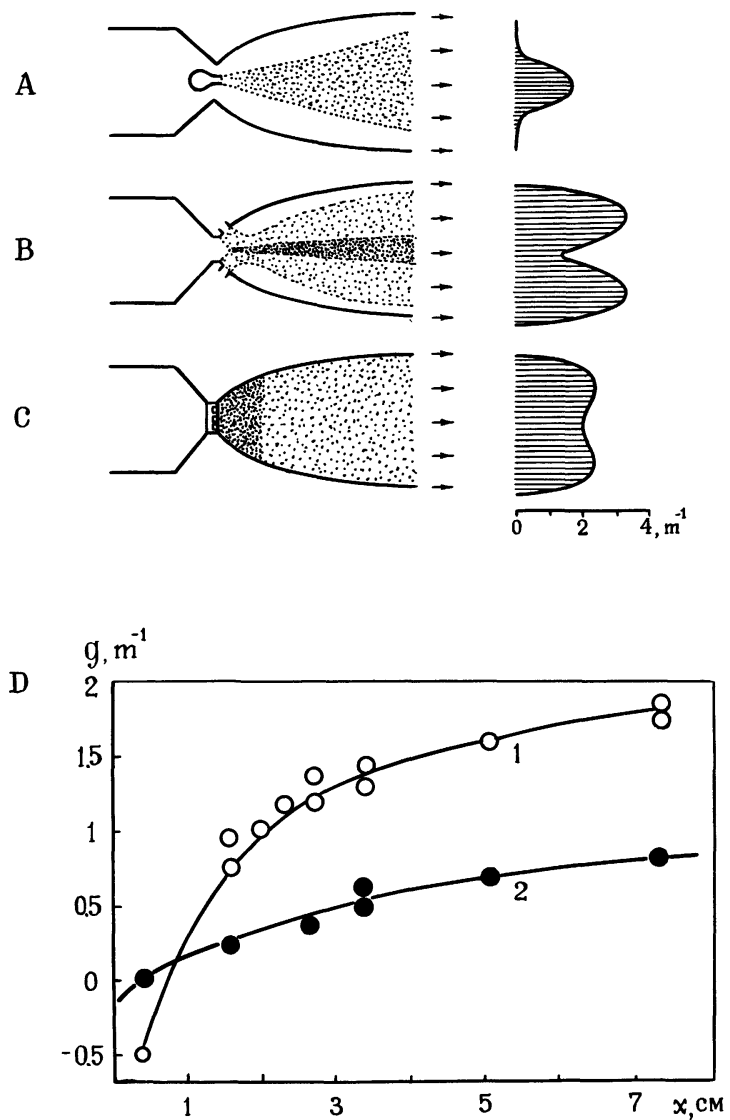

Fig. 15. - Typical profiles of MGDLs gain distribution for different regimes [16] (upper part of the figure) and the gain distribution downstream the nozzle in the mixing regime (1) and in the conventional GDL (2).

The measured values of $g$ versus $T$ for the central part of the flow (variant in Fig. 15B) are given in figure 16. Nitrogen and air served as an exciting gas. The value of $g$ in the premixed gas GDLs measured on the same set-up [14] are plotted in this figure for the sake of comparison. The analysis shows that temperature range in the GDLs with selective excitation attains high values, thus indicating the possibility of obtaining higher efficiencies and available energy in such systems. Figure 16 illustrates the dependence of $g$

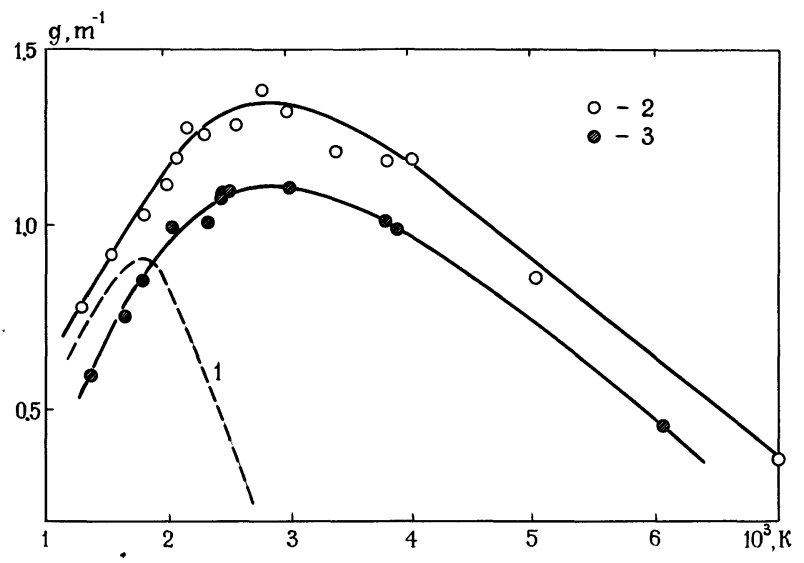

Fig. 16. - Experimental data on temperature dependent gain in the central part of the nozzle : 1 , premixed GDL ; 2,3 , mixing regimes for nitrogen and air (exciting gases), respectively.

on $T$ for a fixed mixing geometry. In this regime the flow temperature at the point of injection varied from 400 to $2000 \mathrm{~K}$, thus determining a substantial increase of vibrational energy losses in the mixing region at a stagnation temperature above $3000 \mathrm{~K}$. The results for the numerical modeling show that optimization of gasdynamic parameters in the mixing region can preserve a high efficiency of vibrational energy freezing to $5000 \mathrm{~K}$. This has been also proved by the experimental data [18] where the gain is above $3 \mathrm{~m}^{-1}$ at $T=5000 \mathrm{~K}$ in the MGDLs.

It should be borne in mind that these high temperatures increase the gain due to hot transitions [53]. This effect on the resonance radiation absorption at $10.6 \mu \mathrm{m}$ in $\mathrm{CO}_{2}$ has been in details investigated [49]. Figure 17 gives the limited estimates of the gain in the MGDLs depending on a vibrational temperature for different transitions.

The analysis of the data obtained shows that at excitation temperatures $(T \gtrsim 3000 \mathrm{~K})$ the population of the upper vibrational levels becomes intensive and, consequently, the gain much affected by the transitions $01^{1} 1 \rightarrow 11^{1} 0,02^{0} 1 \rightarrow 12^{\circ} 0,0 n 2 \rightarrow 1 n 1$ where $n=0^{0}, 1^{1}$. The measured values of the gain at transition frequencies of 100-001 show that the hot transition contribution cannot be practically recorded because the frequency of these transitions does not coincide with that of the probing signal at low pressure $(P \lesssim 0.05 \mathrm{~atm})$. In the lasing regime, energy extraction occurs in the passes with a maximum gain (the main transition 001-100 may be considered to be this pass at vibrational temperatures of about $7000 \mathrm{~K}$ ).

3.5 Power Characteristics. - The power generation characteristics of a laser system depend not 


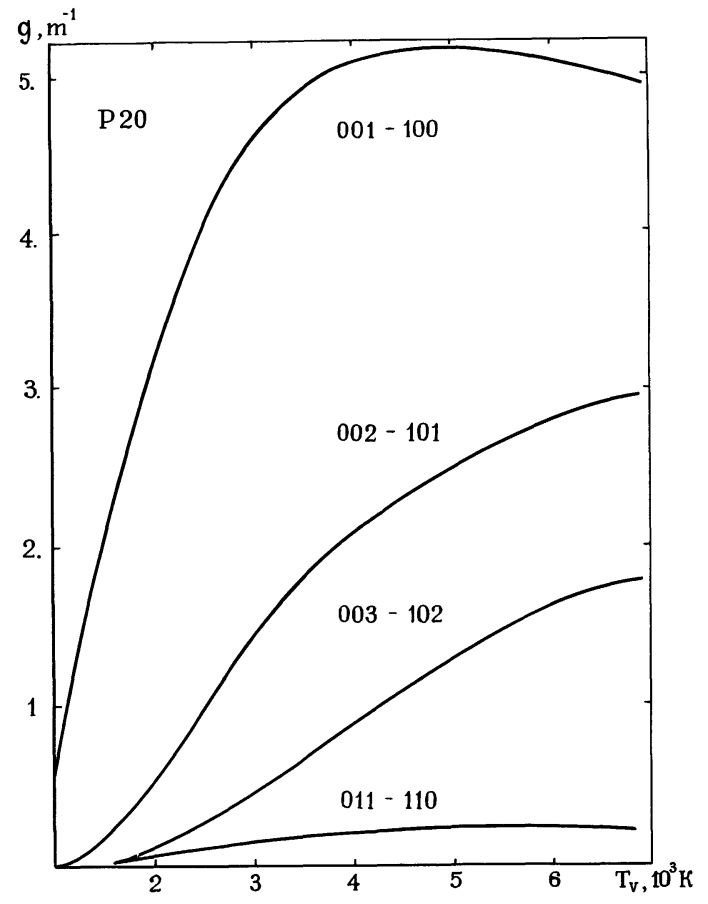

Fig. 17. - Limiting estimates for gain variation with temperature for different transitions. $T_{100}=T^{\prime}=300 \mathrm{~K} ;\left(10 \% \mathrm{CO}_{2}+40 \%\right.$ $\mathrm{N}_{2}+50 \% \mathrm{He}$ ) is the mixture.

only on the properties of a population inversion but, to a great extent, on the optical cavity design and quality. These characteristics of MGDLs have been studied experimentally using different cavities. The length of the active medium varied from $35 \mathrm{~mm}$ $[24,25]$ to $280 \mathrm{~mm}[23]$ and more. In GDLs with selective excitation and supersonic flow mixing the frozen vibrational energy may be effectively extracted in the form of the coherent radiation energy when specific lasing energy approaches that in the electric discharge lasers. For example, in the experiments with arc heating [18] the energy (about $50 \mathrm{~J} / \mathrm{g}$ ) has been recently obtained, however, high specific lasing energies (up to 25-30 J/g) have been achieved by pure thermal excitation in shock waves [14-17], [23-25].

Consider the most important MGDLs power characteristics. Figure 18 is a typical plot of output generation power versus nitrogen stagnation temperature [17]. This confirms the tendency of optimum stagnation temperature to increase with helium content in the injected mixture. The calculated specific lasing energy is about $20 \mathrm{~J} / \mathrm{g}$. A comparison of this value with the numerical modeling and the available energy measured in terms of the gain shows that the specific lasing energy in MGDLs is far from its predicted limit, and the vibrational energy losses during extraction are still of significance. The dynamics of these losses has been studied in [27] by simultaneous measurement of gain in the inflow and outflow areas, the cavity being capable if switching-off with disadjustment of one of the mirrors. These data show that the vibrational energy losses are associated with incomplete

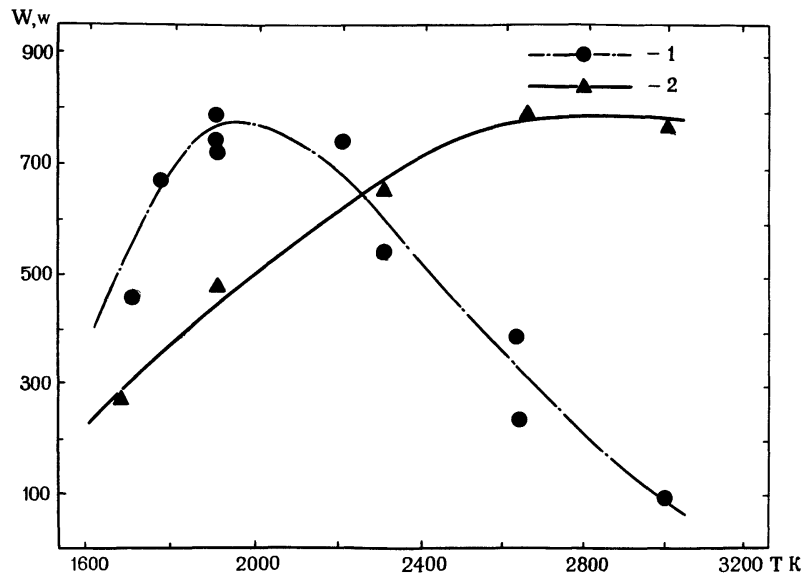

Fig. 18. - Experimental data on laser output power depending on nitrogen stagnation temperature for different ratios of $\mathrm{CO}_{2}$ and $\mathrm{He}$ in the injected mixture : $1, \mathrm{CO}_{2}-\mathrm{He}=1-3 ; 2, \mathrm{CO}_{2}-\mathrm{He}=1-20$. Stagnation pressure $=$ injection pressure $=9 \mathrm{~atm}$.

conversion and unused energy outflow from the switched-on cavity as well as with the effect of flow gasdynamic disturbances and absorption losses in the switched-off cavity.

The typical results on vibrational energy losses are shown in figure 19 [27]. The gain at the cavity entrance (Fig. 19, curve $1, g=(1.7-2) \mathrm{m}^{-1}$ ) satisfies the available vibrational energy (about 80-100 J/g). A decrease in vibrational energy due to only relaxation in typical GDLs at a distance of $10-20 \mathrm{~cm}$ is negligible :
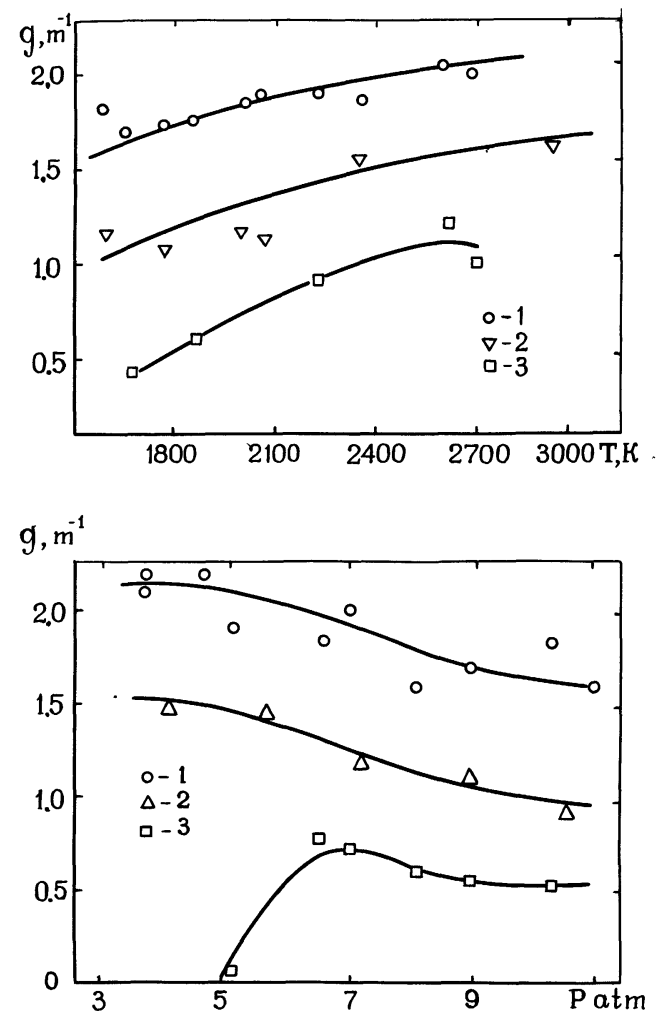

Fig. 19. - Upper part : gain versus nitrogen stagnation temperature at the entrance ( 1$)$ and exit $(2,3)$ of the cavity. (2, the cavity is switched-off, 3, the cavity is loaded.) Lower part : nitrogen stagnation pressure dependence. 
control measurements of the gain fall in the regular gasdynamic flow at a distance of $15 \mathrm{~cm}$ downstream the cavity have shown that vibrational energy losses do not exceed 8-10 J/g. At the same time, the gain fall in the flow through a real resonance switched-off cavity (Fig. 19, curve 2) is substantial. One can assume that there are two possible reasons of decreasing $g$ under these conditions : $a$ ) due to rapid deactivation of the $\mathrm{CO}_{2}$ level .001 in the stagnation pressure and temperature regions ; $b$ ) due to the population of the level 100 with increasing translational temperature in gasdynamic disturbances that originate in the areas of fastening mirrors. With regard for these factors, the total vibrational energy losses (Fig. 19) are 20-35 J/g. As is seen from curve 3 in figure 19, the observed transfer of the vibrational energy out of the switched-on cavity noticeably exceeds these losses and constitutes $30-60 \cdot \mathrm{J} / \mathrm{g}$, depending on the plenum temperature. Thus the estimates show that real losses of the available vibrational energy in the cavity are substantial, and the efficiency of energy conversion into radiation is low, therefore, the improvement of the GDL scheme and of energy extraction in the cavity is still one of the basic problems of designing GDLs. However, in spite of substantial losses and conversion incompleteness, the specific laser energy of MGDLs is rather high, which witnesses promising high efficiency of GDLs with selective excitation.

4. The effect of gasdynamic disturbances and resonance self-absorption on population inversion. - 4.1 GASDYNAMIC DISTURBANCES. - Optical properties of inverted media can, to a great extent, vary in the presence of gasdynamic disturbances : shocks, rarefaction waves, contact surfaces, turbulent wakes, etc. There are different reasons for these disturbance generations : channel wall roughness, separation phenomena, recesses for mirror protection or the use of aerodynamic windows [50-51]. For example, from the gasdynamic estimates [52] it follows that disturbances with a density jump of $\Delta \rho / \rho=4 \%$ are formed at a distance of $10 \mathrm{~cm}$ from the wall step of $0.05 \mathrm{~mm}$ high. Through varying gas temperature and density, the gasdynamic disturbances affect the inversion, promote medium thermalization and cause distortion of beams in the cavity. As can be seen from the above results, the strongest disturbance effects are to be observed in the lasing regime. The gain is estimated depending on varying thermodynamic state of the medium in the disturbance region.

The gain in GDLs is a complex function of temperature, density and population of the $\mathrm{CO}_{2}$ vibrational levels. With gasdynamic disturbances propagating at the initial time instant it may be considered that only thermodynamic properties of a medium vary, while a relative population of vibrational levels remains unchanged since vibrational relaxation occurs slower than the establishment of equilibrium with the translational degrees of freedom. And also the gain in the gasdynamic disturbance at the initial time instant will be, mainly, attributed to varying gas density and rotational sublevels distribution of the $\mathrm{CO}_{2}$ molecules depending on gas temperature. The absolute inversion in this case varies directly proportional to the gas density in the disturbed flow. At subsequent time instants for sufficiently strong disturbances, a gain variation is determined chiefly by varying vibrational level population in the relaxation zone behind a gasdynamic disturbance.

For example, let the frozen flow of inverse medium pass through the rarefaction wave at the Mach number from $M_{\infty}$ to $M$. Then, the varying parameters in the rarefaction wave can be easily determined by the relations for the adiabatic isentropic flow [54] :

$\frac{T}{T_{\infty}}=\frac{1+\frac{\gamma-1}{2} M_{\infty}^{2}}{1+\frac{\gamma-1}{2} M^{2}} ; \quad \frac{P}{P_{\infty}}=\left(\frac{T}{T_{\infty}}\right)^{\gamma / \gamma-1}$

The calculated gains for disturbed states are presented in figure 20.

It is seen from these data that a gain increase in the rarefaction wave due to secondary freezing of partially thermalized mediun is possible only at sufficiently high initial gas densities in the flow. At lower
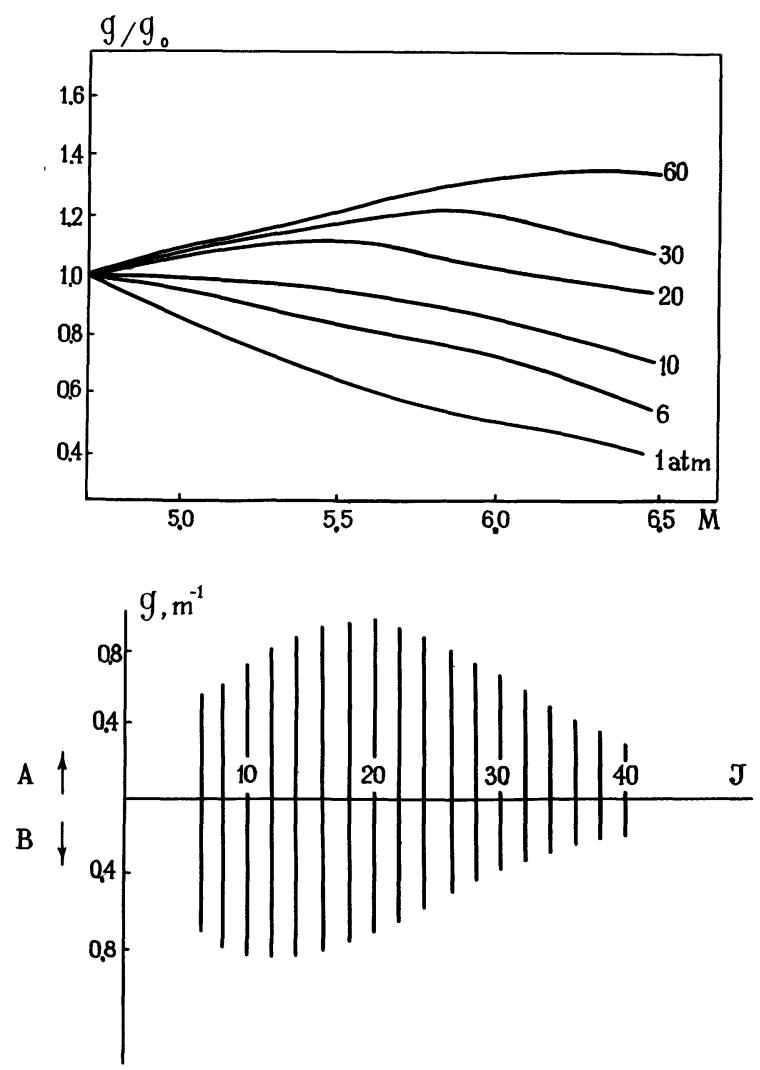

Fig. 20. - Gain coefficient variations in a $\left(\mathrm{CO}_{2}+4 \mathrm{~N}_{2}+5 \mathrm{He}\right)$ mixture in the rarefaction wave depending on the final Mach number for the expanding flow, the initial Mach number being $M=4.7$; $J=18$ (the upper part), and the diagram for the gain distribution over the rotational sublevels before (A) and behind (B) the rarefaction wave (the lower part). 
densities the gain decrease prevails. It should be borne in mind that a variation of certain translational and vibrational temperatures provokes redistribution of effective transitions from different rotational sublevels : with decreasing temperature in the rarefaction wave, maximum gains displace towards the lower rotational quantum numbers. Figure 20 illustrates this effect by a diagram for a gain depending on the rotational quantum number. The gain is calculated for the states before and behind the rarefaction wave. These data (Fig. 20) can be also considered as recommendation for a choice of optimum expansion. The analysis of these data shows that the optimum pressure (with respect to the gain) corresponds to a transition from the Doppler contour to the collisional one with the gain of $0.05 \mathrm{~atm}$ in conventional GDLs.

Consider now the effect of shock waves on population inversion properties [55]. As has already been mentioned, the population of the vibrational level just behind the wave front does not vary (consequently, the vibrational energy concentration remains unchanged), therefore, the gas parameters behind the wave front can be found from the relations for a gas shock with a constant heat capacity ratio $\left(\gamma=c_{\mathrm{p}} / c_{\mathrm{v}}\right)$ [54] :

$$
\frac{P}{P_{\infty}}=\frac{2 \gamma M^{2}-(\gamma-1)}{\gamma+1} ; \frac{T}{T_{\infty}}=\frac{P}{P_{\infty}} \frac{\gamma-1+2 / M^{2}}{\gamma+1} .
$$

The Mach number in these ratios can be calculated in terms of the frozen sound speed. Substituting these expressions into the relations for the gain gives the explicit dependence of the gain behind the shock wave front on the wave Mach number : $g / g_{\infty}=f(M)$.

The numerical results on the varying gain behind the shock front are presented in figure 21. It is seen that there exist three characteristic pressure regions where the gain in shock waves varies differently : at sufficiently small initial pressures $(p \lesssim 0.02 \mathrm{~atm})$ the Doppler broadening of a line prevails and at higher pressures, the collisional one. Simultaneous density and translational gas temperature variations behind the shock front result in increasing $g$ at small pressures (a) when increasing density and absolute, proportional to this growth, inversion are not compensated by the Doppler contour broadening and in decreasing $g$ in the range of the collisional broadening (b). At gas pressure $(p \gtrsim 1 \mathrm{~atm})$ the contribution of the overlapping neighbouring rotational lines to the gain becomes significant; in this case a pressure variation $(p=1-10 \mathrm{~atm})$ is expected to increase the gain in the shock wave. However, as the numerical analysis has shown, in this pressure range the temperature increase becomes more essential than the effect of the overlapping neighbouring rotational lines. Therefore, in the shock wave at a pressure of $p \geqslant 1 \mathrm{~atm}$, the gain also decreases. This effect is however less pronounced than at lower initial pressures.

In the relaxation region behind the shock front, a
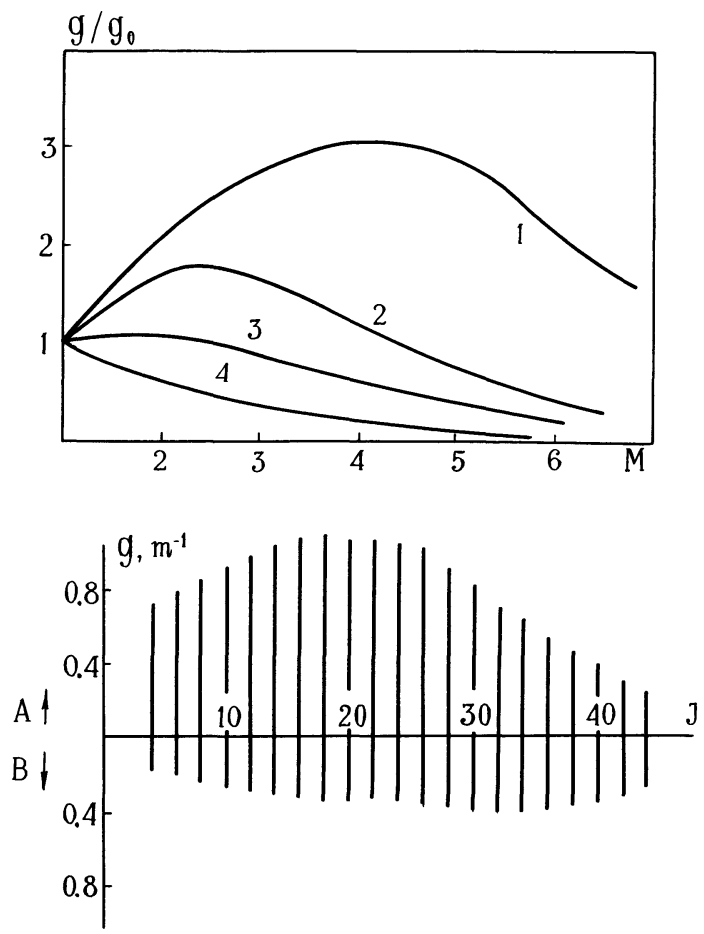

Fig. 21. - Upper part : calculated gain variations in a plane shock wave as a function of the shock Mach number at different initial pressures in the gas flow : $1, P_{0}=0.5 \times 10^{-3} \mathrm{~atm} ; 2 \times 10^{-3}$; $3.2 \times 10^{-3} ; 4.3 \times 10^{-2} . \mathrm{CO}_{2}+4 \mathrm{~N}_{2}+5 \mathrm{He}$ is a working mixture. Lower part : diagram for the gain coefficient distribution over the rotational sublevels before $(\dot{\mathrm{A}})$ and behind (B) the shock wave front. $\mathrm{CO}_{2}+4 \mathrm{~N}_{2}+5 \mathrm{He}$ is a working mixture; $M=4.7$; $P_{\text {in }}=10^{-2}$ atm.

further rapid gain decrease should be expected. This variation also remains for an inclined shock wave, though in this case the gain varies somewhat slower due to the large value of the longitudinal flow component behind the inclined shock wave.

The gasdynamic disturbance effect on the gain has been experimentally checked with a $g$-distribution in the GDL channel provided with a shock tube $\left(5 \times 5 \mathrm{~cm}^{2}\right)$. The gasdynamic disturbances (shock and rarefaction waves) have been artificially generated in the flow using the wedge inserts. Figure 22 gives a schematic of the shock locations and measurements.
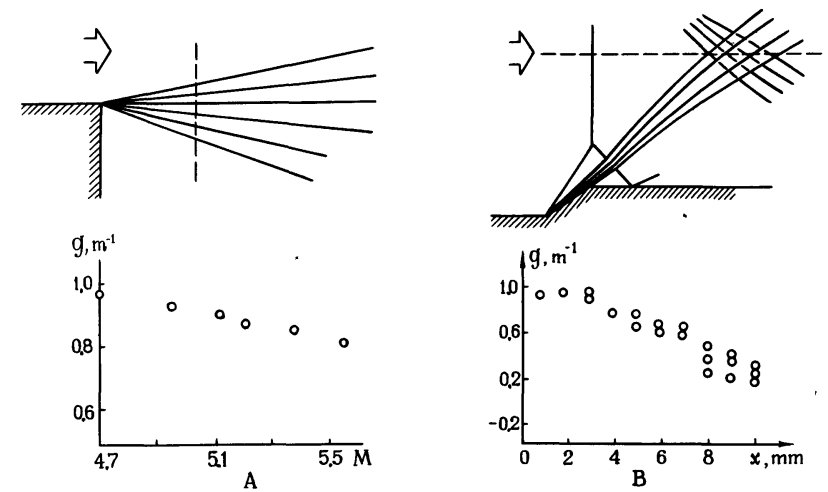

Fig. 22. - Experimental data on varying gain coefficients in steady rarefaction and shock waves. The upper part of the figure illustrates wave flow pattern; the dashed line denotes areas of measurements in the shock tube section. 
The calculated gain of the rarefaction wave past the outer part of the corner (curve 2 corresponds to the experimental conditions $: p=6 \mathrm{~atm}$, figure 20, upper part) and their comparison with the experiment shows a good agreement with prediction. With probing beam displacement downstream the shock wave front (dashed line), the amount of the measured gain decreases monotonically. Similar results have been obtained for the inclined shock waves (with regular crossing or with one insert). Note that in the experiments described, the spatial resolution (with doublepass measuring scheme) is small : $\sim 2 d \simeq 6 \mathrm{~mm}$ where $d$ is the diameter of the probing beam. This resolution is insufficient to record the rapid gain variation behind the shock front. It should be said in conclusion that the experimental data for the effect of gasdynamic disturbances (shock and rarefaction waves) on a spatial distribution and the population inversion in the GDL channel show that the decrease is well described by the simple model, mainly, taking into account the thermodynamic parameters of a medium.

4.2 AbSORPTION PROCESSES. - Simple numerical estimations of the absorption coefficients have been made to define the resonance self-absorption of $10.6 \mu \mathrm{m}$ radiation in the GDL cavity depending on the state parameters. The values of the Einstein coefficient for transitions that define absorption and collisional broadening cross-section are necessary to describe a dependence of the absorptivity on thermal gas dynamic parameters. The data on transition line centers, also needed to calculate the absorption coefficient, are known with a sufficient accuracy for $\mathrm{CO}_{2}$ molecules $[38,39]$.

Based on the analysis of nine independent measurements of the Einstein coefficient $A_{21}$ for P20 line of the transition $10^{\circ} 0 \rightarrow 00^{\circ} 1$, the authors of [57] have recommended the value of $A_{21}=0.187 \mathrm{~s}^{-1}$. This magnitude is a mean value of three most accurate measurements, while the experimental results from [57] give the arithmetic mean of $A_{21}=0.189 \mathrm{~s}^{-1}$, which practically fits the recommended magnitude. The dependence of the Einstein coefficient on the quantum number, $J$, can be defined by expressing $A_{21}$ through the square of the matrix element of the vibrational transition dipole moment $\left(R_{\mathrm{v}^{\prime} \mathrm{v}^{\prime \prime}}\right)^{2}[58]$ :

$A_{21}=\frac{1}{g_{1}} \frac{64 \pi^{4} v_{0}}{3 h c^{3}} S(J) \cdot\left(R_{\mathrm{v}^{\prime} \mathrm{v}^{\prime \prime}}\right)^{2}$,

where $S(J)$ is Hanley-London's factor equal to $J$, with a good accuracy, for the P-branch and $(J+1)$, for the R-branch [58], and $g_{1}$ is the degeneracy factor of the lower state. The tabular data for the optical cross-sections collisional broadening are also presented in [57].

The experimental results [59,60] and [49] have shown that the temperature-dependent absorption coefficient is more accurately described assuming the collisional broadening cross-section depends on temperature in the form of $\sigma \sim T^{-1 / 2}$. In this case at gas temperatures $(T \sim 400-500 \mathrm{~K})$ the contribution of hot transitions $(1 n 0, J) \rightarrow(0 n 1, J \pm 1)$ becomes pronounced where $n=1^{1}, 2^{0}$, as well as of the transitions $(0 n 2, J) \rightarrow(1 n 1, J \pm 1),(0 n 3, J) \rightarrow(1 n 2, J \pm 1)$ where $n=0^{\circ}, 1^{1}$, etc. With account of the hot transitions, the neighbouring line overlap exerts its influence even at pressures $p \sim 1 \mathrm{~atm}$. In [49] the absorption coefficient dependence on medium parameters is described in detail, and figure 23 presents the typical calculation results. A comparison of the predicted and experimental data (Fig. 24) shows that at temperatures

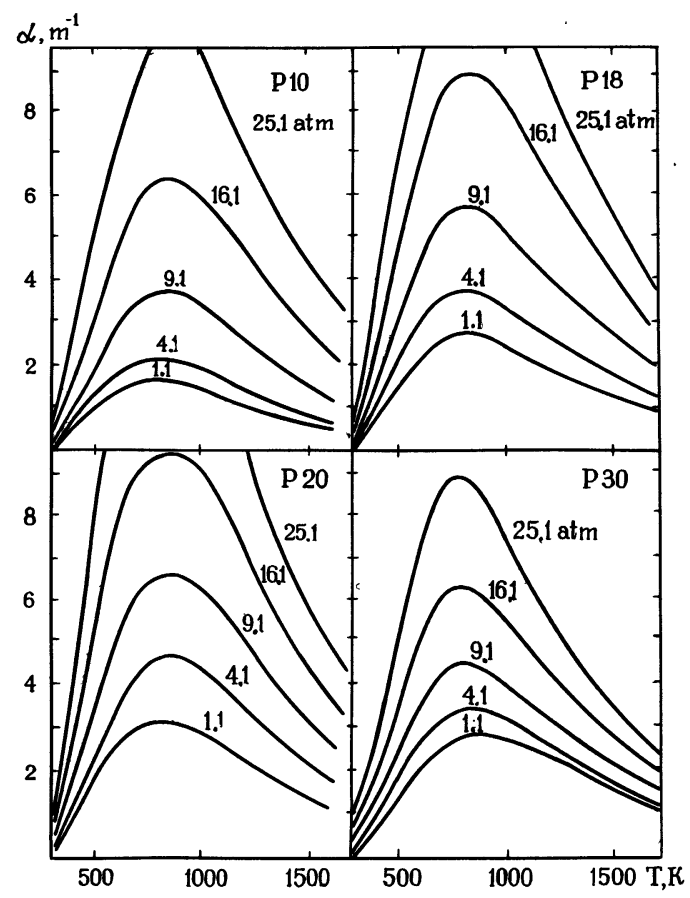

Fig. 23. - Predicted $\mathrm{CO}_{2}$ absorption coefficients versus gas temperature. Gas pressures and rotational lines of the vibrationalrotational transition of the probing laser varied as noted in graphs.

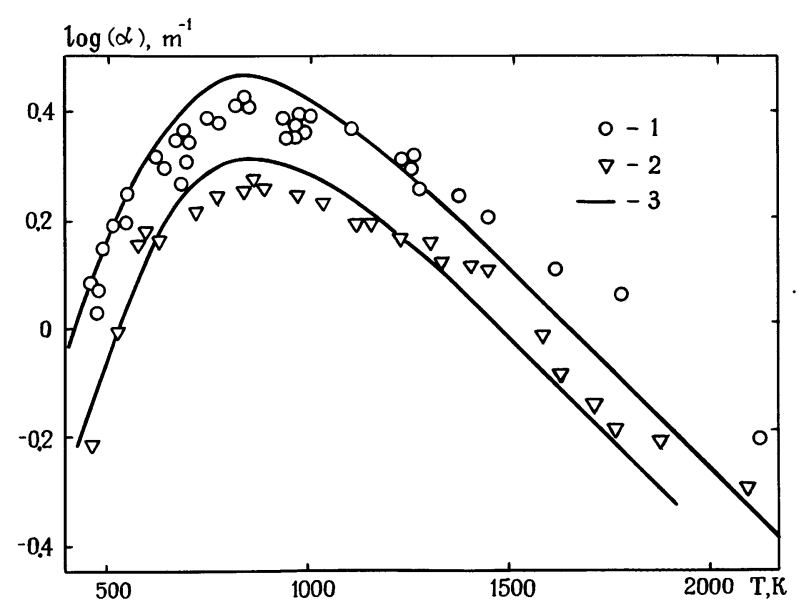

Fig. 24. - Experimental and predicted absorption coefficients of $10.6 \mu \mathrm{m}$ radiation $(\mathrm{P} 18)$ versus gas temperature : 1 , experimental data for $\mathrm{CO}_{2} ; 2$, for $\mathrm{CO}_{2}+\mathrm{N}_{2}$ mixture; 3, prediction. 
up to $T \lesssim 1500 \mathrm{~K}$, a satisfactory agreement is observed. Hence, a knowledge of the value and behaviour of the absorption coefficient varying with gasdynamic flow parameters of the lasing medium makes it possible to estimate the effect of radiation resonance self-absorption by the thermalized zone in the cavity upon GDLs characteristics.

The main parameter in these estimations is the degree of pressure recovery in the stagnation regions. This parameter is affected by the gas thermalization time and absorption coefficient. It can be proved that the absorption is extremely important for the short cavity length lasers when the total gain per one pass is low. Thus, with the use of $10 \% \mathrm{CO}_{2}$ in the laser active medium [16] the total gain per one pass may be expected to decrease from 10 to $30 \%$ in the stagnation region $10 \mathrm{~cm}$ long depending on pressure recovery due to absorption effects. Thus, these estimates point to the necessity of taking into account the vibrational energy losses due to resonance light absorption in the stagnation regions.

5. Conclusions. - Thus, the analysis of the predictions, estimates and experimental data illustrates the substantial effect of flow formation on the optical properties of the laser systems with convective transfer. It is shown that in the systems with selective excitation of a pumping component accompanied by subsequent mixing of the exiting and radiating gases in the supersonic flow, a considerable increase in the gain, specific laser energy and efficiency of available energy conversion into the coherent radiation energy is reached, as compared to the tradition flow development schemes in conventional GDLs. The specific features of modeling of the shocked flow-lasers are discussed and the measurement data are reported on the optical quality of the flow in MGDLs. It is shown that a proper choice of the nozzle design and the way of radiating gas injection into the GDLs with selective excitation permits rapid turbulent mixing of components with small relaxation losses and gives a uniform gas distribution across the channel at drastically high gain coefficients (up to $5 \mathrm{~m}^{-1}$ ).

The measured gains in different cross-sections of the cavity under the switched-off and switched-on conditions define the vibrational energy losses in the GDL cavity that occur due to incomplete conversion and transfer of the unused energy from the cavity and due to gasdynamic disturbances. These results witness that the obtained specific laser energy in the MGDLs is about $50 \mathrm{~J} / \mathrm{g}$. Though this magnitude is drastic for the gasdynamic ways of population inversion, it can be substantially increased by improving the scheme of laser energy extraction from the cavity.

This paper deals also with varying gain in different single gasdynamic disturbances. The experimental data are reported on a significant suppression of gain in the artificially generated gasdynamic disturbances in the GDL channel. Finally, as shown in the last section, the possible effects of the $10.6 \mu \mathrm{m}$ radiation self-absorption on GDL characteristics are essential, and the laser energy losses in the gas stagnation region due to resonance absorption must be accounted of. To conclude, a general note should be done that, the GDLs with double-step freezing when $\mathrm{CO}_{2}$ is injected into the supersonic nitrogen flow, attain drastically high gains, specific available laser energy and rather high efficiency. A further development of the mixed-flow gasdynamic laser concept and technology can promote the improvement of the molecular high-power laser system characteristics.

Acknowledgments. - The authors are indebted to V. N. Croshko, Yu. A. Yacobi, V. K. Konyukhov and S. A. Losev for their many valuable discussions and contributions.

\section{References}

[1] Basov, N. G. and Oraevsky, A. N., Sov. JETP 44 (1963) 1742.

[2] Hurle, J. R. and Hertzberg, A., Phys. Fluids 8 (1965) 1601.

[3] Konyukhov, V. K. and Prokhorov, A. M., Sov. JETPPis'ma 3 (1966) 436.

[4] Basov, N. G., Mikhailov, V. G., Oraevsky, A. N. and Shcheglov, V. A., Sov. J. Techn. Phys. 38 (1968) 2031.

[5] Basov, N. G., Oraevsky, A. N. and Shcheglov, V. A., Sov. Journ. Tech. Phys. 37 (1967) 339.

[6] Wisnievski, E. E., Fein, M. E., Verdeyen, J. T. and CherringTON, B. E., Appl. Phys. Lett. 12 (1968) 257.

[7] Fein, M. E., Verdeyen, J. T. and Cherrington, B. E., Appl. Phys. Lett. 14 (1969) 337.

[8] Bronfin, B. R., Boedeker, L. R. and Chayer, J. P., Appl. Phys. Lett. 16 (1970) 214.

[9] Croshko, V. N., Soloukhin, R. I. and Wolanski, P., Opt. Commun. 6 (1972) 275.

[10] Croshko, V. N., Soloukhin, R. I. and Wolanski, P., Recent Developments in Shock Tube Research, Proc. 9th Shock Tube Symp., Eds. D. Berchader and W. Griffith (Stanford Univ. Press) 1973, p. 167.
[11] Croshko, V. N. and Soloukhin, R. I., Dokl. AN SSSR 211 (1973) 829.

[12] Taran, J. P. E., Charpenel, M. and Borghi, R., AIAA-Paper No. 73-622 (1973).

[13] Taran, J. P. E., Charpenel, M. and Borghi, R., Appl. Phys. Lett. 2 (1973) 661

[14] Croshko, V. N., Soloukhin, R. I. and Fomin, N. A., Fiz. Goreniya Vzryva 10 (1974) 473.

[15] Croshko, V. N., Soloukhin, R. I. and Fomin, N. A., Mixed Flow Gasdynamic Laser, in : Gas Lasers (Nauka Publ. Novosibirsk) (1977), p. 59.

[16] Croshko, V. N., Fomin, N. A. and Soloukhin, R. I., Acta Astron. 2 (1975) 929.

[17] Crauklis, A. V., Croshko, V. N., Soloukhin, R. I. and Fomin, N. A., Fiz. Goreniya Vzryva 12 (1976) 792.

[18] Bailly, R., Pealat, M. and Taran, J. P. E., Revue Phys. Appl. 12 (1977) 1705.

[19] Milewski, J., Brunne, M., Stanco, J., Zielinski, A., IrCZUK, M., Kusmierek, I., Bull. Acad. Pol. Sci., Ser. Sci. Techn. 20 (1972) 73. 
[20] Milewski, J., Brunne, M., Irczuk, M., Stanco, J., Zielinski, A., Rabczuk, G., Demin, A. I., Kudryavtsev, E. M., Volkov, A. Yu. and Sobolev, N. N., Appl. Phys. Lett. 30 (1977) 342.

[21] Kasuya, K., Kumasaka, T. and Murasaki, T., Japan. J. Appl. Phys. 12 (1973) 771.

[22] Kasuya, K., Migamoto, M., Horioka, K., Fujiwara, K. and Murasaki, T., Japan. J. Appl. Phys. 15 (1976) 1397.

[23] Gassady, P., Newton, J. and Rose, P., AiAA-Paper No. 76343 (1976).

[24] Schall, W., Hoffmann, P., Huegel, H., J. Appl. Phys. 48 (1977) 688 .

[25] Hoffman, P., Hugel, H., Schall, W., AIAA-Journal 15 (1977) 1527.

[26] Volkov, A. Yu., Demin, A. I. and Crauklis, A. V., Preprint No. 189, P. N. Lebedev Physics Inst., Moscow, 1977.

[27] Crauklis, A. V., Croshko, V. N., Soloukhin, R. I. and Fomin, N. A., in : Kinetics of Chemical Reactions (Institute of Chemical Physics, Chernogolovka) 1977.

[28] Borghi, R. and Charpenel, M., Astronautica Acta 17 (1972) 833.

[29] Breev, V. V., Kukharenko, A. G., Napartovich, A. P. and Sharkov, V. F., Teplofiz. Vysok. Temp. 12 (1974) 1325.

[30] Achasov, O. V., Soloukhin, R. I., Fomin, N. A., in : Heat and Mass Transfer Problems, p. 89, Heat and Mass Transfer Institute (Minsk) 1977.

[31] Ostroukhov, N. N. and TKAChenKo, V. K., Kvantovaya Electron. 5 (1978) 924.

[32] Diakov, A. S., Didyukov, A. I., Tkachenko, V. K. and Cherkasov, E. M., Kvantovaya Electron. 5 (1978) 1166.

[33] Russell, D. A., Astronaut. Aeronaut. 6 (1975) 50.

[34] Taylor, R. L., Bitterman, S., Rev. Mod. Phys. 41 (1969) 26.

[35] Christiansen, W. H. and Tsongas, G. A., Phys. Fluids 14 (1971) 2611.

[36] Buonadonna, V. R. and Chistiansen, W. H., Recent Development in Shock Tube Research, Proc. 9th Int. Shock Tube Symp. (Eds. D. Bershader, W. Griffith) (Stanford Univ. Press, 1973), p. 173.

[37] Biryukov, A. S. and Gordiets, B. F., Zh. Prikl. Mekh. Tekhn. Fiz. 6 (1972) 29.

[38] BiRyukov, A. S., in : Theory of Spectroscopy and Gasdynamic Lasers, Proc. P. N. Lebedev Physics Institute 83 (1975) 13
[39] ANDerson, J. D., Gasdynamic Lasers : An Introduction (Academic Press, N.Y.) 1976.

[40] Losev, S. A., Gasdynamic Lasers (Nauka Publ., Moscow) 1977.

[41] Volkov, A. Yu., Demin, A. I., Logunov, A. N., KudryavtSEv, E. M. and Sobolev, N. N., Preprint No. 4 (P. N. Lebedev Physics Institute, Moscow) 1977.

[42] Vandysheva, N. M., Leningrad Univ. Herald 13 (1976) 57.

[43] Kuehn, D. M. and Monson, D. J., Appl. Phys. Lett. 16 (1970) 48.

[44] 'Dronov, A. P., Kudryavtsev, E. M., Sobolev, N. N., Diakov, A. S., Sov. JETP-Pis'ma 11 (1970) 516.

[45] SoloukHIN, R. I., Shock Tube in Flow Laser Research : Modeling and Applications, in : Proc. 11th Intern. Shock Tube Symp. (Eds. B. Ahlborn, A. Hertzberg and D. Russel) (Washington Univ. Press., Seattle and London) 1978.

[46] Croshko, V. N., Soloukhin, R. I. and Fomin, N. A., Fiz. Goreniya Vzryva 9 (1973) 352.

[47] Oppenheim, A. K., Urtiew, P. A. and Laderman, A. J., Archiwum Budowy Maszyn (Warszawa) 11 (1964) 441.

[48] SмiтH, C. E., J. Fluid Mech. 25 (1966) 625.

[49] Soloukhin, R. I. and Fomin, N. A., Zh. Prikl. Mekh. Tekhn.' Fiz. 1 (1977) 42.

[50] Parmentier, E. M. and Greenberg, R. A., AiAA-Journal 11 (1973) 943.

[51] Hertzberg, A., AIAA-Paper No. 77-83, 1977 ; also : J. Energy 1 (1977) 331.

[52] Russell, D. A., AIAA-Paper, No. 74-223, 1974.

[53] Bertel, I. M., Kuntsevich, B. F., Petukhov, V. O., Trushin, S. A. and Churakov, V. V., Zh. Prikl. Spectrosc. 28 (1978) 804.

[54] LorTsansky, L. G., Mechanics of Fluids and Gases (Nauka, Publ., Moscow) 1978.

[55] Kozlov, G. I. and Stuprtsky, E. L., Sov. J. Techn. Phys. 45 (1975) 359.

[56] Soloukhin, R. I. and Fomin, N. A., Dokl. AN SSSR 228 (1976) 3, 596.

[57] Biryukov, A. S., Volkov, A. Yu., Kudryavtsev, E. M. and SERIKOv, R. I., Kvantovaya Electron. 3 (1976) 1748.

[58] HerTZBerg, G., Vibrational and Rotational Spectra of Multiatomic Molecules (Izd. Inostr. Lit. Moscow) 1949.

[59] Ely, R. and MCCubBin, T. K., Appl. Opt. 9 (1970) 1230.

[60] Strilchuk, A. R. and Offenderger, A. A., Appl. Opt. 13 (1974) 2643. 